\title{
Matched interface and boundary (MIB) method for elliptic problems with sharp-edged interfaces
}

\author{
Sining Yu ${ }^{\text {a }}$, Yongcheng Zhou ${ }^{\text {a }}$, G.W. Wei ${ }^{\text {a,b,* }}$ \\ ${ }^{a}$ Department of Mathematics, Michigan State University, D301 WH, East Lansing, MI 48824, USA \\ ${ }^{\mathrm{b}}$ Department of Electrical and Computer Engineering, Michigan State University, D301 WH, East Lansing, MI 48824, USA
}

Received 3 May 2006; received in revised form 13 October 2006; accepted 17 October 2006

Available online 13 December 2006

\begin{abstract}
Elliptic problems with sharp-edged interfaces, thin-layered interfaces and interfaces that intersect with geometric boundary, are notoriously challenging to existing numerical methods, particularly when the solution is highly oscillatory. This work generalizes the matched interface and boundary (MIB) method previously designed for solving elliptic problems with curved interfaces to the aforementioned problems. We classify these problems into five distinct topological relations involving the interfaces and the Cartesian mesh lines. Flexible strategies are developed to systematically extends the computational domains near the interface so that the standard central finite difference scheme can be applied without the loss of accuracy. Fictitious values on the extended domains are determined by enforcing the physical jump conditions on the interface according to the local topology of the irregular point. The concepts of primary and secondary fictitious values are introduced to deal with sharp-edged interfaces. For corner singularity or tip singularity, an appropriate polynomial is multiplied to the solution to remove the singularity. Extensive numerical experiments confirm the designed second order convergence of the proposed method.
\end{abstract}

(c) 2006 Elsevier Inc. All rights reserved.

Keywords: Immersed boundary method; Immersed interface method; Ghost fluid method; Elliptic equations; Sharp-edged interfaces

\section{Introduction}

Since the pioneer work of Peskin [50] in 1977, much attention has been paid to the numerical solution of elliptic equations with discontinuous coefficients and singular sources on regular Cartesian grids $[7,8,11,15,18,20,30-32,55,58]$. Simple Cartesian grids are preferred in these studies since the complicated procedure of generating unstructured grid could be bypassed, and well developed fast algebraic solvers could be utilized. The importance of elliptic interface problems has been well recognized in a variety of disciplines, such as fluid dynamics [16,19,29,47], electromagnetics [23,24] and material science [26]. However, to construct

\footnotetext{
* Corresponding author. Address: Department of Mathematics, Michigan State University, D301 WH, East Lansing, MI 48824, USA. Tel.: +1 517353 4689; fax: +1 5174321562 .

E-mail address: wei@math.msu.edu (G.W. Wei).
} 
highly efficient methods for these problems is a difficult task due to the low global regularity of the solution. Traditional numerical methods that are constructed with the assumption of smooth solutions cannot perform at designed accuracy, and might even diverge. For this class of problems, apart from Peskin's immersed boundary method (IBM) [21,33,50-52], a number of other elegant methods have been proposed. Among them, the immersed interface method (IIM), proposed by LeVeque and $\mathrm{Li}$ [35] is a second order sharp interface scheme. The IIM has been made robust and efficient over the past decade [1,14,36,37,54]. The ghost fluid method (GFM) [17] was proposed as a relatively simple and easy to use approach. For irregular interfaces, it is nature to construct a solution in the finite element method formulation $[2,9,38]$, in particular, using the discontinuous Galerkin technique [22]. A relevant, while quite distinct approach is the integral equation method for complex geometry $[44,45]$. Aforementioned methods have found much success in scientific and engineering applications [6-8,15,18,20,25-28,30,32,34,39,41,40,42,53,54,57-59]. A possible further direction in the field could be the development of higher order interface methods $[20,60,61]$ which are particularly desirable for problems involving both material interfaces and high frequency oscillations, such as the interaction of turbulence and shock, and high frequency wave propagation in inhomogeneous media [5].

One of the most challenging problems in the field is the solution of elliptic equations with sharp-edged coefficients, i.e., non-smooth interfaces. Numerical solutions to this class of problems have widespread applications in science and engineering, such as electromagnetic wave scattering and propagation $[12,48,49]$, wave-guides analysis [46], plasma-surface interaction [43], friction modeling [56] and turbulent-flow [4]. To the best of our knowledge, none of the aforementioned methods proposed for elliptic interface problems have been directly applied to the treatment of sharp-edged interfaces. Essentially, as the gradient near the tips of sharp-edged interface is not well defined, some earlier interface methods might not work. Most existing results on this class of problems are obtained by using finite element methods $[46,49]$. However, finite element methods might exhibit a reduced convergence rate when used for the analysis of geometries containing sharp edges $[25,49]$. Consequently, dramatic local mesh refinement is required in the vicinity of sharp edges [13], and leads to severe increase in computational time and memory requirement. In particular, local mesh refinement does not work if the solution is highly oscillatory due to the so-called pollution effect [3], which is a common situation in dealing with electromagnetic wave scattering and propagation. Hou and Liu proposed a finite element formulation [25] for solving elliptic equations with sharp-edged interfaces. Remarkably, these authors have achieved about 0.8 th order convergence with non-body-fitting grids.

The objective of the present work is to extend the matched interface and boundary (MIB) method previously designed for solving elliptic problems with curved interfaces to problems with sharp-edged interfaces, thin-layered interfaces and interfaces that intersect with the geometric boundary. The MIB was proposed by Zhao and Wei [60] as a systematic higher-order method for electromagnetic wave propagation and scattering in dielectric media. Recently, it has been generalized for solving elliptic equations with curved interfaces by Zhou et al. [61]. The MIB approach makes use of fictitious domains so that the standard high order central finite difference (FD) method can be applied across the interface without the loss of accuracy. The fictitious values on fictitious domains are determined from enforcing the interface jump conditions at the exact position of the interface. One feature of the MIB is that it disassociates between the discretization of the elliptic equation and the enforcement of interface jump conditions. Another feature is to make repeated use of the lowest order jump conditions to determine the fictitious values on extended domains. Since only lowest order interface jump conditions are repeatedly used in the MIB method, arbitrarily high order convergence can be achieved in principle. For straight interfaces, MIB schemes of up to 16th order have been constructed $[60,61]$. For lightly curved interfaces, up to 6 th order schemes have been demonstrated [61]. Most recently, we have proposed an interpolation formulation of the MIB method without the explicit use of fictitious values [62]. We have shown that our interpolation formulation is equivalent to our earlier fictitious domain formulation. Fourth order convergence is obtained for arbitrarily curved interfaces. In the present work, we further generalize the MIB method to allow the presence of sharp-edged interfaces, thin-layered interfaces and interfaces that intersect with the domain boundary. For these problems, flexible strategies that have not been ever considered before are required. We introduce the concepts of primary and secondary fictitious values to overcome the difficulty of sharp-edged interfaces. The essence is to replace unavailable auxiliary points by secondary fictitious points to resolve primary fictitious values when there are geometric difficulties. The topological relations between the interfaces and the Cartesian mesh lines are classified into five distinct types. For each 
topology, appropriate secondary fictitious values, auxiliary points and jump conditions will be selected. In this work, the classification procedure and the solution scheme are made systematic and automatic. The generalized MIB method is designed to have 2nd order accuracy even if the interface is Lipschitz continuous while not $C^{1}$. It captures sharp kinks at the interface without a priori knowledge of these kinks. The problem becomes more difficult as the interface edge is getting sharper. For an acute angle that is larger than the critical value $2 \tan ^{-1}\left(\frac{1}{3}\right)$, the present method can handle with the designed 2 nd order convergence. However, edges with their acute angles smaller than the critical value can also be treated to the 2 nd order accuracy if the mesh lines that bisect edges are not vertical or horizontal, which is true in most problems. For the problems with smooth (say $C^{1}$ ) interfaces, the present method can easily achieve 2 nd order convergence since required auxiliary points can always be found by refining the mesh.

It is well known that Galerkin formulations can directly solve problems with weak solution [10,25]. Whereas, collocation formulations cannot directly handle this class of problems. Nevertheless, a standard technique is to multiply the solution with an appropriate polynomial factor [49] to remove the singularity. A new equation can be derived and solved. Then, it is matter of algebraic operation to resolve the original solution. This approach is incorporated in the present method to deal with problems with weak solutions.

The rest of this paper is organized as follows. In Section 2, we introduce the generalized MIB method that is able to treat sharp-edged interfaces, thin-layered interfaces and interfaces that intersect with geometric boundary. The critical angle of the sharp edge is analyzed. Both on-interface and off-interface schemes are proposed to handled five different topological relations. In Section 3, the proposed MIB method is validated for a wide variety of problems, ranging from those with critical small angles, edge tip on a grid point, highly oscillatory solution, multiple edges, multiply connected interfaces, a missile geometry, thin-layered coatings and a weak solution. This paper ends with a conclusion.

\section{Theory and algorithm}

In this section, we briefly describe the mathematical problem. Irregular points that are on the interface are treated differently from those off the interface. A pseudo-code is provided for the present method. We analyze the critical acute angle that can be treated by the proposed method.

\subsection{Basic problem}

Consider an open bounded domain $\Omega \subset R^{2}$. Let $\Gamma$ be the interface which divides $\Omega$ into disjoint open subdomains, $\Omega^{+}$and $\Omega^{-}$, hence $\Omega=\Omega^{+} \cup \Omega^{-} \cup \Gamma$. Assume that the boundary $\partial \Omega$ and interface $\Gamma$ are Lipschitz continuous and there is a piecewise smooth level-set function $\phi$ on $\bar{\Omega}$, which $\Gamma=\{\phi=0\}, \Omega^{-}=\{\phi<0\}$ and $\Omega^{+}=\{\phi>0\}$. We seek solutions of the $2 \mathrm{D}$ elliptic equation with variable diffusion coefficient $\beta(x, y)$ away from the interface $\Gamma$ given by

$$
\left(\beta(x, y) u(x, y)_{x}\right)_{x}+\left(\beta(x, y) u(x, y)_{y}\right)_{y}=q(x, y), \quad x \in \Omega \backslash \Gamma .
$$

Consider a case where the interface intersects the $j$ th mesh line at a point which is between $(i, j)$ and $(i+1, j)$. A direct calculation of $u_{x x}$ at $(i, j)$ using the second-order central difference scheme involves grid points $u_{i-1, j}, u_{i, j}$ and $u_{i+1, j}$ and will lead to the reduction in the convergence order. A solution to this problem is to replace $u_{i+1, j}$ at the irregular point $(i+1, j)$ by a fictitious value $f_{i+1, j}$, which is a smooth extension of function values from the left hand side of the interface. A jump condition is required to uniquely determine $f_{i+1, j}$. For the same reason, $(i+1, j)$ is also an irregular point and $f_{i, j+1}$ is a fictitious value required for the central finite difference scheme at $(i+1, j)$.

For elliptic equation (1), the solution might admit a prescribed jump at an interface point. Moreover, the gradient along the normal direction can also be prescribed. Therefore, two jump conditions can be given

$$
\begin{aligned}
& {[u]=u^{+}-u^{-}=\varphi,} \\
& {\left[\beta u_{n}\right]=\beta^{+} u_{n}^{+}-\beta^{-} u_{n}^{-}=\psi,}
\end{aligned}
$$


where normal vector $\vec{n}=(\cos \theta, \sin \theta)$ can be defined a.e. on $\Gamma$ and points from $\Omega^{-}$to $\Omega^{+}$, while $0 \leqslant \theta<2 \pi$ is the angle between positive $x$-direction and the vector $\vec{n}$. We assume that both $[u]$ and $\left[\beta u_{n}\right]$ are $C^{1}$ continuous along the Lipschitz continuous interface $\Gamma$. When considering the interface which is not always aligned with the $x$ - or $y$ - mesh line, one more interface condition can be attained by differentiating Eq. (2) along the tangential direction of the interface, $\vec{\tau}=(-\sin \theta, \cos \theta)$. Hence for a point $\left(x_{0}, y_{0}\right)$ on the interface, we have three jump conditions,

$$
\begin{aligned}
& {[u]=u^{+}-u^{-}=\varphi,} \\
& {\left[u_{\tau}\right]=\left(-u_{x}^{+} \sin \theta+u_{y}^{+} \cos \theta\right)-\left(-u_{x}^{-} \sin \theta+u_{y}^{-} \cos \theta\right)=\rho,} \\
& {\left[\beta u_{n}\right]=\beta^{+}\left(u_{x}^{+} \cos \theta+u_{y}^{+} \sin \theta\right)-\beta^{-}\left(u_{x}^{-} \cos \theta+u_{y}^{-} \sin \theta\right)=\psi .}
\end{aligned}
$$

To enforce these jump conditions, we must compute $u_{x}^{+}, u_{x}^{-}, u_{y}^{+}$and $u_{y}^{-}$. For a given interface geometry, it can be very difficult to compute some of these four partial derivatives. One way to avoid this difficulty is to use only two of three jump conditions Eqs. (4)-(6). Therefore we can eliminate one of four partial derivatives, $u_{x}^{+}, u_{x}^{-}, u_{y}^{+}$and $u_{y}^{-}$, and result in the following sets of jump conditions:

$$
[u]=u^{+}-u^{-}, \quad \text { and } \quad\left[\beta u_{n}\right]-\beta^{-} \tan \theta\left[u_{\tau}\right]=C_{x}^{+} u_{x}^{+}-C_{x}^{-} u_{x}^{-}+C_{y}^{+} u_{y}^{+},
$$

where $C_{x}^{+}=\beta^{+} \cos \theta+\beta^{-} \tan \theta \sin \theta, C_{x}^{-}=\beta^{-} \cos \theta+\beta^{-} \tan \theta \sin \theta$ and $C_{y}^{+}=\beta^{+} \sin \theta-\beta^{-} \sin \theta$;

$$
[u]=u^{+}-u^{-}, \quad \text { and } \quad\left[\beta u_{n}\right]-\beta^{+} \tan \theta\left[u_{\tau}\right]=C_{x}^{+} u_{x}^{+}-C_{x}^{-} u_{x}^{-}-C_{y}^{-} u_{y}^{-},
$$

where $C_{x}^{+}=\beta^{+} \cos \theta+\beta^{+} \tan \theta \sin \theta, C_{x}^{-}=\beta^{-} \cos \theta+\beta^{+} \tan \theta \sin \theta$ and $C_{y}^{-}=\beta^{-} \sin \theta-\beta^{+} \sin \theta$;

$$
[u]=u^{+}-u^{-}, \quad \text { and } \quad\left[\beta u_{n}\right]+\beta^{-} \cot \theta\left[u_{\tau}\right]=C_{x}^{+} u_{x}^{+}+C_{y}^{+} u_{y}^{+}-C_{y}^{-} u_{y}^{-},
$$

where $C_{x}^{+}=\left(\beta^{+}-\beta^{-}\right) \cos \theta, C_{y}^{+}=\beta^{-} \cos \theta \cot \theta+\beta^{+} \sin \theta$ and $C_{y}^{-}=\beta^{-}(\cos \theta \cot \theta+\sin \theta)$, and

$$
[u]=u^{+}-u^{-} \quad \text { and } \quad\left[\beta u_{n}\right]+\beta^{+} \cot \theta\left[u_{\tau}\right]=C_{x}^{-} u_{x}^{-}+C_{y}^{+} u_{y}^{+}-C_{y}^{-} u_{y}^{-},
$$

where $C_{x}^{-}=\left(\beta^{+}-\beta^{-}\right) \cos \theta, C_{y}^{-}=\beta^{+} \cos \theta \cot \theta+\beta^{-} \sin \theta$ and $C_{y}^{+}=\beta^{+}(\cos \theta \cot \theta+\sin \theta)$. For given local environment, only one of these four combinations is needed to determine two fictitious values. We choose an appropriate combination such that its involved partial derivatives can be conveniently computed.

To restore the order of convergence of the discretization at irregular points, we need to treat $\left(\beta u_{x}\right)_{x}$ and $\left(\beta u_{y}\right)_{y}$ to the designed order of convergence near the interface. Since these two terms are considered separately, we only need to illustrate how to locally recover the second order accuracy of the standard 3-point central FD scheme for $\left(\beta u_{x}\right)_{x}$. The treatment of $\left(\beta u_{y}\right)_{y}$ can be carried out similarly. We classify irregular points as off-interface ones and on-interface ones. Different schemes are required to deal with these cases.

\subsection{Off-interface scheme}

Off-interface scheme considers the situation that a pair of adjacent irregular points are separated by the interface and neither of them stays on the interface. As shown in Fig. 1, the intersection of the interface $\Gamma$ and the $x$ mesh line is $\left(x_{0}, y_{0}\right)$. We discuss the interface schemes at irregular point $(i, j) \in \Omega^{+}$and irregular point $(i+1, j) \in \Omega^{-}$. At each irregular point, we need to construct a primary fictitious value. A primary fictitious value is a value that will be used in the standard finite difference discretization. While a secondary fictitious value is one that helps resolving a primary fictitious value. However, the secondary fictitious value can also be a primary fictitious value for the discretization of other irregular points. The concept of secondary fictitious values is essential for overcoming the geometric difficulties created by sharp-edged interfaces.

\subsubsection{Off-interface scheme 1}

Off-interface scheme 1 is the standard MIB scheme for the situation that the curvature of the interface is small and there is no sharp edge. In this situation, two primary fictitious values can be solved together without the use of secondary fictitious values. Define auxiliary point as the point that is next to the irregular point and 


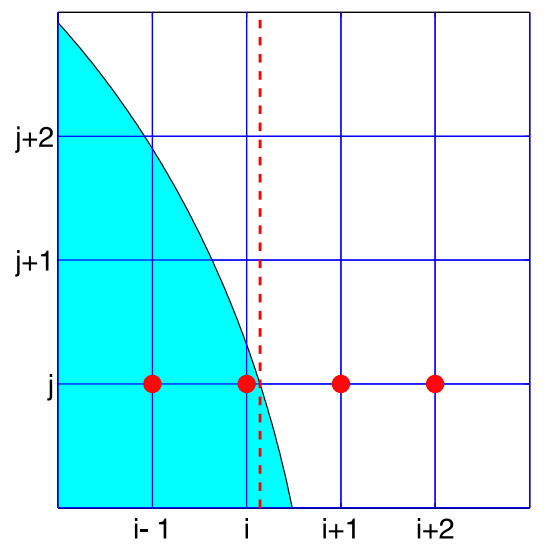

Fig. 1. Situation handled by Off-interface scheme 1. $(i-1, j),(i+2, j)$ with respect to the present irregular points.

stays on the mesh line that connects two irregular points and crosses the interface. Therefore, there are two auxiliary points for each pair of off-interface irregular points. When curvature is relatively small or there is no sharp edge, both auxiliary points belong to a 'proper' region. A proper region refers to a neighborhood where inside each subdomain, $\Omega^{+}$or $\Omega^{-}$, at least one auxiliary point can be found next to each irregular point. This situation is shown in Fig. 1. To discretize $\left(\beta u_{x}\right)_{x}$ by standard second order FD central scheme at irregular point $(i, j)$, primary fictitious value $f_{i+1, j}$ is needed. Similarly, primary fictitious value $f_{i, j}$ is needed for the discretization at irregular point $(i+1, j)$. Since both auxiliary points belong to the 'proper' region, standard MIB scheme can be used to solve for these two primary fictitious values without use of a secondary fictitious value. We consider jump conditions at the intersection between the interface and the $j$ th mesh line. In this particular case, it is convenient to use jump conditions (8) since they involve $u^{+}, u^{-}, u_{x}^{+}, u_{x}^{-}$and $u_{y}^{-}$at the intersection point $\left(x_{0}, y_{0}\right)$. Here $u^{+}$and $u^{-}$are obtained by interpolations from information in $\Omega^{+}$and $\Omega^{-}$, respectively. $u_{x}^{+}$is computed from $u_{i-1, j}, u_{i, j}$ and $f_{i+1, j}$. Similarly, $u_{x}^{-}$is computed from $f_{i, j}, u_{i+1, j}$ and $u_{i+2, j}$. These expressions are explicitly given as follows:

$$
\begin{aligned}
& u^{+}=\left(w_{0, i-1}, w_{0, i}, w_{0, i+1}\right) \cdot\left(u_{i-1, j}, u_{i, j}, f_{i+1, j}\right)^{\mathrm{T}}, \\
& u^{-}=\left(w_{0, i}, w_{0, i+1}, w_{0, i+2}\right) \cdot\left(f_{i, j}, u_{i+1, j}, u_{i+2, j}\right)^{\mathrm{T}}, \\
& u_{x}^{+}=\left(w_{1, i-1}, w_{1, i}, w_{1, i+1}\right) \cdot\left(u_{i-1, j}, u_{i, j}, f_{i+1, j}\right)^{\mathrm{T}}, \\
& u_{x}^{-}=\left(w_{1, i}, w_{1, i+1}, w_{1, i+2}\right) \cdot\left(f_{i, j}, u_{i+1, j}, u_{i+2, j}\right)^{\mathrm{T}},
\end{aligned}
$$

where $w_{n, m}$ denote finite difference (FD) weights. The first subscript $n$ represents either interpolation $n=0$ or first order derivative $n=1$ at interface point $\left(x_{0}, y_{0}\right)$, while their second subscript is for the node index.

To approximate $u_{y}^{-}$, we need three $u$ values in the $y$-direction. These values are located at intersection points between the dash line and $j$ th, $(j+1)$ th and $(j+2)$ th mesh line, see Fig. 1. Unfortunately, these values are unavailable and have to be approximated from interpolation schemes along the $x$-direction. This means six more auxiliary points are involved. In practice, one of these two can be easily found. In the situation shown in Fig. 1, $u_{y}^{-}$can be represented as follows:

$$
\begin{aligned}
u_{y}^{-}= & {\left[w_{1, j}, w_{1, j+1}, w_{1, j+2}\right] \cdot\left[\begin{array}{ccccccccc}
w_{0, i} & w_{0, i+1} & w_{0, i+2} & 0 & 0 & 0 & 0 & 0 & 0 \\
0 & 0 & 0 & w_{0, i}^{\prime} & w_{0, i+1}^{\prime} & w_{0, i+2}^{\prime} & 0 & 0 & 0 \\
0 & 0 & 0 & 0 & 0 & 0 & w_{0, i-1}^{*} & w_{0, i}^{*} & w_{0, i+1}^{*}
\end{array}\right] } \\
& \times\left[f_{i, j}, u_{i+1, j}, u_{i+2, j}, u_{i, j+1}, u_{i+1, j+1}, u_{i+2, j+1}, u_{i-1, j+2}, u_{i, j+2}, u_{i+1, j+2}\right]^{\mathrm{T}} .
\end{aligned}
$$

Substituting Eqs. (11) and (12) into jump condition Eq. (8), we have

$$
[u]=\left(w_{0, i-1}, w_{0, i}, w_{0, i+1}\right) \cdot\left(u_{i-1, j}, u_{i, j}, f_{i+1, j}\right)^{\mathrm{T}}-\left(w_{0, i}, w_{0, i+1}, w_{0, i+2}\right) \cdot\left(f_{i, j}, u_{i+1, j}, u_{i+2, j}\right)^{\mathrm{T}},
$$




$$
\begin{aligned}
& {\left[\beta u_{n}\right]-\beta^{+} \tan \theta\left[u_{\tau}\right]=C_{x}^{+}\left[\begin{array}{c}
w_{1, i-1} \\
w_{1, i} \\
w_{1, i+1}
\end{array}\right]^{\mathrm{T}} \cdot\left[\begin{array}{c}
u_{i-1, j} \\
u_{i, j} \\
f_{i+1, j}
\end{array}\right]-C_{x}^{-}\left[\begin{array}{c}
w_{1, i} \\
w_{1, i+1} \\
w_{1, i+2}
\end{array}\right]^{\mathrm{T}} \cdot\left[\begin{array}{c}
f_{i, j} \\
u_{i+1, j} \\
u_{i+2, j}
\end{array}\right]} \\
& -C_{y}^{-}\left[\begin{array}{c}
w_{1, j} \\
w_{1, j+1} \\
w_{1, j+2}
\end{array}\right]^{\mathrm{T}}\left[\begin{array}{ccccccccc}
w_{0, i} & w_{0, i+1} & w_{0, i+2} & 0 & 0 & 0 & 0 & 0 & 0 \\
0 & 0 & 0 & w_{0, i}^{\prime} & w_{0, i+1}^{\prime} & w_{0, i+2}^{\prime} & 0 & 0 & 0 \\
0 & 0 & 0 & 0 & 0 & 0 & w_{0, i-1}^{*} & w_{0, i}^{*} & w_{0, i+1}^{*}
\end{array}\right] \\
& \times\left[f_{i, j}, u_{i+1, j}, u_{i+2, j}, u_{i, j+1}, u_{i+1, j+1}, u_{i+2, j+1}, u_{i-1, j+2}, u_{i, j+2}, u_{i+1, j+2}\right]^{\mathrm{T}} .
\end{aligned}
$$

Solving for $f_{i, j}$ and $f_{i+1, j}$ from Eqs. (13) and (14), then the representation of these fictitious values in terms of function values and the physical jumps can be written as

$$
\begin{aligned}
& f_{i, j}=C^{i} \cdot U, \\
& f_{i+1, j}=C^{i+1} \cdot U,
\end{aligned}
$$

where $C^{i}=\left(C_{1}^{i}, C_{2}^{i}, \ldots, C_{13}^{i}\right)$ and $C^{i+1}=\left(C_{1}^{i+1}, C_{2}^{i+1}, \ldots, C_{13}^{i+1}\right)$ are the expansion coefficients of two fictitious values with respect to 10 function values and 3 jumps which are also given by the vector $U=\left(u_{i-1, j}, u_{i, j}, u_{i+1, j}\right.$, $\left.u_{i+2, j}, u_{i, j+1}, u_{i+1, j+1}, u_{i+2, j+1}, u_{i-1, j+2}, u_{i, j+2}, u_{i+1, j+2},[u],\left[\beta u_{n}\right],\left[u_{\tau}\right]\right)^{\mathrm{T}}[61]$.

With these two expansions of $f_{i, j}$ and $f_{i+1, j}$, one could discretize $\left(\beta u_{x}\right)_{x}$ at irregular points $(i, j)$ and $(i+1, j)$ as if at a regular point:

$$
\begin{aligned}
& \left(\beta u_{x}\right)_{x}=\frac{1}{\Delta x^{2}}\left(\beta_{i-\frac{1}{2}, j}^{+},-\beta_{i-\frac{1}{2}, j}^{+}-\beta_{i+\frac{1}{2}, j}^{+}, \beta_{i+\frac{1}{2}, j}^{+}\right) \cdot\left(u_{i-1, j}, u_{i, j}, f_{i+1, j}\right)^{\mathrm{T}} \quad \text { at }(i, j), \\
& \left(\beta u_{x}\right)_{x}=\frac{1}{\Delta x^{2}}\left(\beta_{i+\frac{1}{2}, j}^{-},-\beta_{i+\frac{1}{2}, j}^{-}-\beta_{i+\frac{3}{2}, j}^{-}, \beta_{i+\frac{3}{2}, j}^{-}\right) \cdot\left(f_{i, j}, u_{i+1, j}, u_{i+2, j}\right)^{\mathrm{T}} \quad \text { at }(i+1, j) .
\end{aligned}
$$

To find the fictitious values at a pair of irregular points $(i, j)$ and $(i+1, j)$, auxiliary points $(i-1, j)$ and $(i, j)$ must be on the same of side of the interface and auxiliary points $(i+2, j)$ and $(i+1, j)$ must also be on the same side of the interface. However, when the interface has a large curvature or a sharp corner, or one of the auxiliary points is out of computational domain, these conditions can no longer be satisfied. The other two offinterface schemes are presented below to deal with these situations.

\subsubsection{Off-interface scheme 2}

Off-interface scheme 2 is designed for sharp-edged interfaces. It is very common in this situation that one cannot locate one auxiliary point for each irregular point inside a subdomain. For example, as depicted in Fig. 2, irregular point $(i, j)$ has no auxiliary point associated with it inside the same subdomain. This 'improper' region cannot be eliminated by refining the mesh. The same situation will occur if the mesh is doubled in both
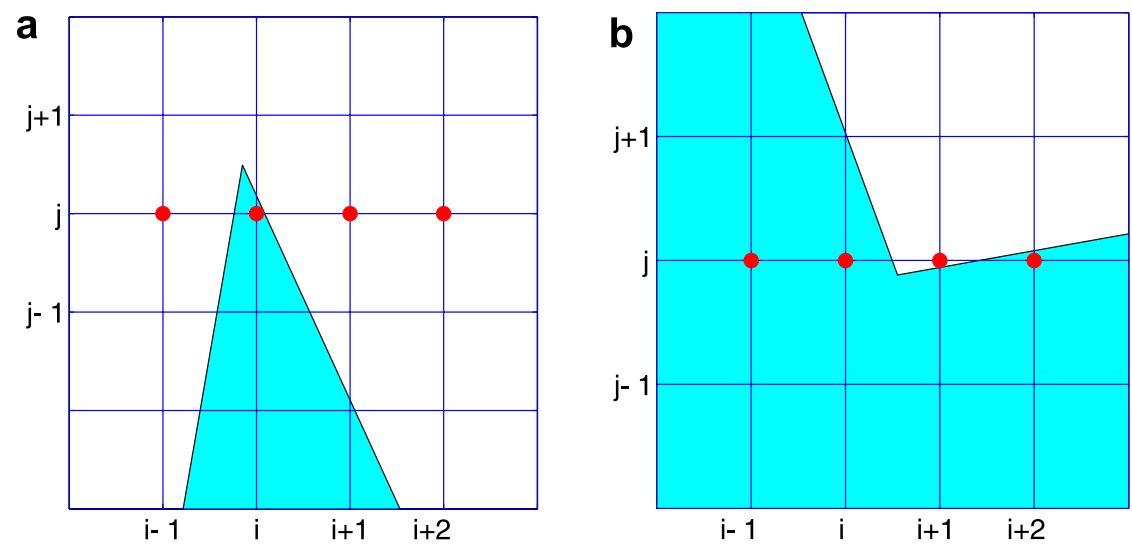

Fig. 2. Situation (a) handled by Off-interface scheme 2(a); Situation (b) handled by Off-interface scheme 2(b). 
the $x$-direction and the $y$ direction. This difficulty is not resolvable by the previous MIB scheme because the auxiliary points in the 'improper' region leads to inconsistence between the number of unknowns and the number of jump conditions. In this case, we introduce the secondary fictitious values, $f_{i-1, j}$ and $f_{i+2, j}$, to replace the role of the auxiliary points in the 'improper' region. Therefore the number of unknowns can be reduced, and the resulting unknowns can be solved by Off-interface scheme 2(a) or 2(b).

2.2.2.1. Off-interface scheme 2(a). Let $F=\left[F_{i, j}, F_{i+1, j}, F_{i-1, j}, F_{i+2, j}\right]^{\mathrm{T}}$, where $F_{i, j}=f_{i, j}$ and $F_{i+1, j}=f_{i+1, j}$ are primary fictitious values at irregular points $(i, j)$ and $(i+1, j)$. If $(i-1, j)$ and $(i, j)$ belong to the same subdomain, $F_{i-1, j}$ represents function value $u_{i-1, j}$. Otherwise, $F_{i-1, j}$ is the secondary fictitious value $f_{i-1, j}$ at point $(i-1, j)$. Similarly, $F_{i+2, j}=u_{i+2, j}$ if $(i+1, j)$ and $(i+2, j)$ belong to the same subdomain. Otherwise, $F_{i+2, j}$ is the secondary fictitious value $f_{i+2, j}$ at point $(i+2, j)$. The expansion (15) could be rewritten as

$$
B \cdot F=\widetilde{C} \cdot \widetilde{U},
$$

where

$$
\begin{aligned}
B & =\left[\begin{array}{cccc}
1 & 0 & -C_{1}^{i} & -C_{4}^{i} \\
0 & 1 & -C_{1}^{i+1} & -C_{4}^{i+1}
\end{array}\right], \\
\widetilde{C} & =\left[\begin{array}{ccccc}
C_{2}^{i} & C_{3}^{i} & C_{5}^{i} & \cdots & C_{13}^{i} \\
C_{2}^{i+1} & C_{3}^{i+1} & C_{5}^{i+1} & \cdots & C_{13}^{i+1}
\end{array}\right], \\
\widetilde{U} & =\left[u_{i, j}, u_{i+1, j}, u_{i, j+1}, u_{i+1, j+1}, u_{i+2, j+1}, u_{i-1, j+2}, u_{i, j+2}, u_{i+1, j+2},[u],\left[\beta u_{n}\right],\left[u_{\tau}\right]\right]^{\mathrm{T}} .
\end{aligned}
$$

If two of $F, F_{m}$ and $F_{l}$, are function values or secondary fictitious values that have already been expressed in terms of function values, while the other two components of $F, F_{i}$ and $F_{j}$, are the fictitious values whose expansions are to be determined, Eq. (18) can be rewritten as

$$
\left[\begin{array}{ll}
B_{1, i} & B_{1, j} \\
B_{2, i} & B_{2, j}
\end{array}\right] \cdot\left[\begin{array}{l}
F_{i} \\
F_{j}
\end{array}\right]=\widetilde{C} \cdot \widetilde{U}-\left[\begin{array}{ll}
B_{1, l} & B_{1, m} \\
B_{2, l} & B_{2, m}
\end{array}\right] \cdot\left[\begin{array}{l}
F_{l} \\
F_{m}
\end{array}\right],
$$

with $i, j, l, m \in\{1,2,3,4\}$. Therefore,

$$
\left[\begin{array}{l}
F_{i} \\
F_{j}
\end{array}\right]=\left[\begin{array}{ll}
B_{1, i} & B_{1, j} \\
B_{2, i} & B_{2, j}
\end{array}\right]^{-1} \cdot\left[\widetilde{C} \cdot \widetilde{U}-\left[\begin{array}{ll}
B_{1, l} & B_{1, m} \\
B_{2, l} & B_{2, m}
\end{array}\right] \cdot\left[\begin{array}{l}
F_{l} \\
F_{m}
\end{array}\right]\right]
$$

gives the expression of $F_{i}$ and $F_{j}$. With obtained primary fictitious values, $\left(\beta u_{x}\right)_{x}$ can be discretized at point $(i, j)$ and $(i+1, j)$ as Eq. (17).

Notice that the expansion of fictitious value $f_{i, j}$ is not unique. For example, as shown in Fig. 2(a), points $(i, j+1),(i+1, j)$ and $(i-1, j)$ are all in $\Omega^{-}$and $f_{i, j}$ should have three expansions by considering three different pairs of points, $(i, j)$ and $(i, j+1),(i, j)$ and $(i+1, j)$, and $(i, j)$ and $(i-1, j)$. Each expansion can be generated by the jump condition at the intersection point of the interface and the grid line segment between a pair of points. These three expansions should be stored separately if they are all available. In the present work, different expansions of a fictitious value are labeled by directions, $\{x+, x-, y+, y-\}$. Here $x+$ means the expansion is generated by the jump condition involving points $(i, j)$ and $(i+1, j), x$ - involving $(i, j)$ and $(i-1, j)$, $y+$ involving $(i, j)$ and $(i, j+1)$ and $y$ - involving $(i, j)$ and $(i, j-1)$. When the primary fictitious value is needed for the standard five point FD scheme, the expansion generated by the nearest pair of points should be used. For example, the five point FD scheme at point $(i, j+1)$ should select the expansion of $f_{i, j}$ from $y+$ direction, while the five point FD scheme of point $(i+1, j)$ should choose the expansion of $f_{i, j}$ from $x+$ direction. If this cannot be done, the expansion from $y+$ direction can be carried out instead. A simple rule is to always select the expansion generated by the nearest pair of points to achieve the best accuracy.

2.2.2.2. Off-interface scheme 2(b). If only one of $F$ component, $F_{i}(i \in\{1,2,3,4\})$, is the fictitious value whose expansion is to be determined and the other three are function values or have expansions in term of function values, then the jump condition of $u(x, y)$ is enough for the determination of $F_{i}$ : 


$$
u^{+}-u^{-}=[u] .
$$

Let $w_{0}^{+}=\left(w_{0, i-1}^{+}, w_{0, i}^{+}, w_{0, i+1}^{+}\right)$and $w_{0}^{-}=\left(w_{0, i}^{-}, w_{0, i+1}^{-}, w_{0, i+2}^{-}\right)$. Then Eq. (24) can be written as

$$
w_{0}^{+} \cdot\left(F_{i-1, j}, u_{i, j}, F_{i+1, j}\right)^{\mathrm{T}}-w_{0}^{-} \cdot\left(F_{i, j}, u_{i+1, j}, F_{i+2, j}\right)^{\mathrm{T}}=[u] .
$$

Rearranging the above equation, we have

$$
B^{\prime} \cdot F=C^{\prime} \cdot U^{\prime}
$$

where $B^{\prime}=\left(-w_{0, i}^{-}, w_{0, i+1}^{+}, w_{0, i-1}^{+},-w_{0, i+2}^{-}\right), C^{\prime}=\left(-w_{0, i}^{+}, w_{0, i+1}^{-}, 1\right)$ and $U^{\prime}=\left(u_{i, j}, u_{i+1, j},[u]\right)^{\mathrm{T}}$.

Solving for $F_{i}$ from the above equation, we have

$$
F_{i}=B_{i}^{\prime-1}\left(C^{\prime} \cdot U^{\prime}-\sum_{l=1, l \neq i}^{4} B_{l}^{\prime} F_{l}\right) .
$$

Eq. (27) gives the expansion of $F_{i}$ in terms of function values since $F_{l}$ are function values or have expansions in terms of function values. With this expansion, $\left(\beta u_{x}\right)_{x}$ can be discretized at point $(i, j)$ as in Eq. (17).

\subsection{On-interface scheme}

\subsubsection{On-interface scheme 1}

On-interface scheme 1 is the standard MIB scheme for the situation that one of primary irregular points is on interface. Two primary fictitious values along the $x$ - and $y$-directions are solved together. Therefore two pairs of irregular points on $x$ - and $y$-directions are involved at the same time. That is, there are a total of three irregular points, one on interface, one in the $x$-direction and the other in the $y$-direction. On-interface scheme 1 requires two auxiliary points and two off-interface irregular points all belong to the same subdomain. Fig. 3 shows a typical situation that can be resolved by On-interface scheme 1 . Note that when $[u] \neq 0$ the function value on the interface is not well defined. From the computational point of view the interface itself can be regarded either as part of the interior or as part of the exterior. Here we regard $u(i, j)=u^{+}(i, j)$ if a grid point $(i, j)$ is on the interface.

Two primary fictitious values are required in order to formulate the difference scheme for $\left(\beta u_{x}\right)_{x}+\left(\beta u_{y}\right)_{y}$ at point $(i, j)$. As indicated in Fig. 3, if points $(i-2, j),(i-1, j),(i, j-1)$ and $(i, j-2)$ belong to subdomain $\Omega^{-}$, the approximations for all derivatives, $u_{x}^{+}, u_{x}^{-}, u_{y}^{+}$and $u_{y}^{-}$are available. Therefore Eqs. (5) and (6) exactly provide two approximate equations for $f_{i, j-1}$ and $f_{i-1, j}$

$$
\begin{aligned}
& {\left[u_{\tau}\right]=(-\sin \theta, \cos \theta, \sin \theta,-\cos \theta) \cdot\left(u_{x}^{+}, u_{y}^{+}, u_{x}^{-}, u_{y}^{-}\right)^{\mathrm{T}}} \\
& {\left[\beta u_{n}\right]=\left(\beta^{+} \cos \theta, \beta^{+} \sin \theta,-\beta^{-} \cos \theta,-\beta^{-} \sin \theta\right) \cdot\left(u_{x}^{+}, u_{y}^{+}, u_{x}^{-}, u_{y}^{-}\right)^{\mathrm{T}},}
\end{aligned}
$$

with

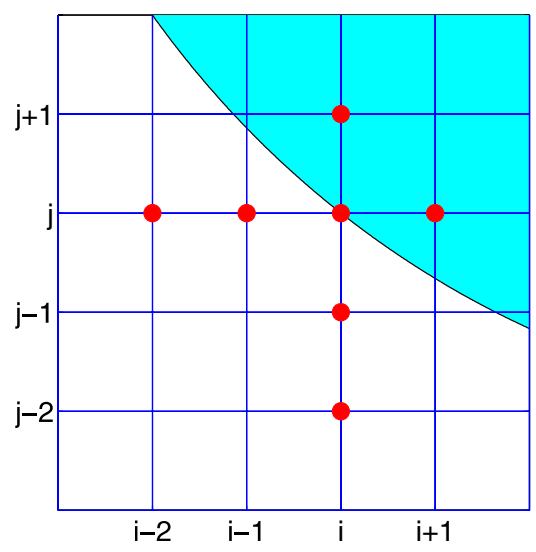

Fig. 3. Situation handled by On-interface scheme 1 . 


$$
\begin{aligned}
& u_{x}^{+}=\left(w_{1, i-1}, w_{1, i}, w_{1, i+1}\right) \cdot\left(f_{i-1, j}, u_{i, j}, u_{i+1, j}\right)^{\mathrm{T}}, \\
& u_{x}^{-}=\left(w_{1, i-2}^{\prime}, w_{1, i-1}^{\prime}, w_{1, i}^{\prime}\right) \cdot\left(u_{i-2, j}, u_{i-1, j}, u_{i, j}-[u]\right)^{\mathrm{T}}, \\
& u_{y}^{+}=\left(w_{1, j-1}, w_{1, j}, w_{1, j+1}\right) \cdot\left(f_{i, j-1}, u_{i, j}, u_{i, j-1}\right)^{\mathrm{T}}, \\
& u_{y}^{-}=\left(w_{1, j-2}^{\prime}, w_{1, j-1}^{\prime}, w_{1, j}^{\prime}\right) \cdot\left(u_{i, j-2}, u_{i, j-1}, u_{i, j}-[u]\right)^{\mathrm{T}} .
\end{aligned}
$$

Substituting Eqs. (30), (31) into jump conditions (28) and (29), and solving for $f_{i, j-1}$ and $f_{i-1, j}$, the expansions of $f_{i, j-1}$ and $f_{i-1, j}$ in terms of real unknowns and jump conditions at point $(i, j)$ can be obtained [61]. Then the difference scheme of the Poisson equation at point $(i, j)$ can be written in $\Omega^{+}$:

$$
\begin{aligned}
\left(\beta u_{x}\right)_{x}+\left(\beta u_{y}\right)_{y}= & \frac{1}{\Delta x^{2}}\left(\beta_{i-\frac{1}{2}, j}^{+},-\beta_{i-\frac{1}{2}, j}^{+}-\beta_{i+\frac{1}{2}, j}^{+}, \beta_{i+\frac{1}{2}, j}^{+}\right) \cdot\left(f_{i-1, j}, u_{i, j}, u_{i+1, j}\right)^{\mathrm{T}} \\
& +\frac{1}{\Delta y^{2}}\left(\beta_{i, j-\frac{1}{2}}^{+},-\beta_{i, j-\frac{1}{2}}^{+}-\beta_{i, j+\frac{1}{2}}^{+}, \beta_{i, j+\frac{1}{2}}^{+}\right) \cdot\left(f_{i, j-1}, u_{i, j}, u_{i, j+1}\right)^{\mathrm{T}} .
\end{aligned}
$$

In some situation, auxiliary points $(i-2, j)$ and $(i, j-2)$ do not belong to $\Omega^{-}$. Instead, we have points $(i+2, j)$ and $(i, j+2)$ in $\Omega^{+}$. The other two primary fictitious values $f_{i+1, j}$ and $f_{i, j+1}$ need to be considered instead. The approximations for all derivatives, $u_{x}^{+}, u_{x}^{-}, u_{y}^{+}$and $u_{y}^{-}$are given as

$$
\begin{aligned}
& u_{x}^{+}=\left(w_{1, i}, w_{1, i+1}, w_{1, i+2}\right) \cdot\left(u_{i, j}, u_{i+1, j}, u_{i+2, j}\right)^{\mathrm{T}}, \\
& u_{x}^{-}=\left(w_{1, i-1}^{\prime}, w_{1, i}^{\prime}, w_{1, i+1}^{\prime}\right) \cdot\left(u_{i-1, j}, u_{i, j}-[u], f_{i+1, j}\right)^{\mathrm{T}}, \\
& u_{y}^{+}=\left(w_{1, j}, w_{1, j+1}, w_{1, j+2}\right) \cdot\left(u_{i, j}, u_{i, j+1}, u_{i, j+2}\right)^{\mathrm{T}}, \\
& u_{y}^{-}=\left(w_{1, j-1}^{\prime}, w_{1, j}^{\prime}, w_{1, j+1}^{\prime}\right) \cdot\left(u_{i, j-1}, u_{i, j}-[u], f_{i, j+1}\right)^{\mathrm{T}} .
\end{aligned}
$$

Substituting Eqs. (33) and (34) into Eqs. (28) and (29), and solving for $f_{i+1, j}$ and $f_{i, j+1}$, the expansions of the primary fictitious unknowns can be obtained and the difference scheme of the Poisson equation at point $(i, j)$ can therefore be written in $\Omega^{-}$:

$$
\begin{aligned}
\left(\beta u_{x}\right)_{x}+\left(\beta u_{y}\right)_{y}= & \frac{1}{\Delta x^{2}}\left(\beta_{i-\frac{1}{2}, j}^{-},-\beta_{i-\frac{1}{2}, j}^{-}-\beta_{i+\frac{1}{2}, j}^{-}, \beta_{i+\frac{1}{2}, j}^{-}\right) \cdot\left(u_{i-1, j}, u_{i, j}-[u], f_{i+1, j}\right)^{\mathrm{T}} \\
& +\frac{1}{\Delta y^{2}}\left(\beta_{i, j-\frac{1}{2}}^{-},-\beta_{i, j-\frac{1}{2}}^{-}-\beta_{i, j+\frac{1}{2}}^{-}, \beta_{i, j+\frac{1}{2}}^{-}\right) \cdot\left(u_{i, j-1}, u_{i, j}-[u], f_{i, j+1}\right)^{\mathrm{T}} .
\end{aligned}
$$

One of two conditions must be satisfied in this scheme: points $(i-2, j)$ and $(i, j-2) \in \Omega^{-}$, or points $(i+2, j)$ and $(i, j+2) \in \Omega^{+}$. In a situation that meets none of these two conditions, On-interface scheme 2 is needed.

\subsubsection{On-interface scheme 2}

As mentioned above, the On-interface scheme 1 requires that two auxiliary points and two irregular points all belong to the same subdomain. However, for concave or multiply connected geometry, this requirement is hardly satisfied. On-interface scheme 2 can handle a situation that has two irregular points while without auxiliary points on the same subdomain. The present scheme utilizes formulas in the $x$-direction and in the $y$-direction separately to resolve secondary fictitious values first. Then the primary fictitious values are solved. The situation is illustrated in Fig. 4, where auxiliary point $(i, j-2) \in \Omega^{-}$while $(i+2, j) \in \Omega^{+}$. The secondary fictitious values $f_{i+1, j}$ and $f_{i, j-1}$ can be obtained. However, since the fictitious values $f_{i, j+1}$ and $f_{i-1, j}$ are not available, the Poisson equation at point $(i, j)$ can neither be solved in $\Omega^{+}$by Eq. (32) nor in $\Omega^{-}$by Eq. (35). Therefore, the secondary fictitious values $f_{i+1, j}$ and $f_{i, j-1}$ are used to solve primary fictitious values $f_{i, j+1}$ and $f_{i-1, j}$.

First, in order to obtain the secondary fictitious values $f_{i+1, j}$ and $f_{i, j-1}$, Eq. (33) are used to obtain $u_{x}^{+}$and $u_{x}^{-}$. Eq. (31) is used to obtain $u_{y}^{+}$and $u_{y}^{-}$. Substituting this equation into Eqs. (28) and (29) and solving for $f_{i+1, j}$ and $f_{i, j-1}$, the representation of secondary fictitious values in terms of real values can be written as

$$
\left[\begin{array}{l}
f_{i+1, j} \\
f_{i, j-1}
\end{array}\right]=\left[\begin{array}{l}
C^{i+1} \\
C^{j-1}
\end{array}\right] \cdot U,
$$

where $C^{i+1}, C^{j-1}$ are the expansion coefficient vectors, and 


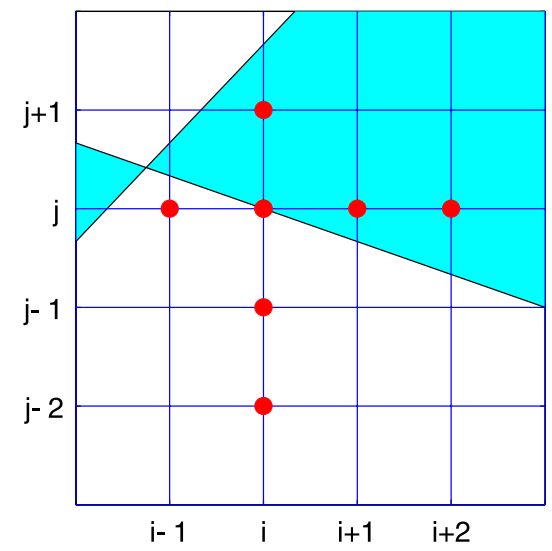

Fig. 4. Situation handled by On-interface scheme 2.

$$
U=\left(u_{i, j}, u_{i-1, j}, u_{i, j-1}, u_{i+1, j}, u_{i, j+1},[u],\left[u_{\tau}\right],\left[\beta u_{n}\right]\right)^{\mathrm{T}} .
$$

Second, the primary fictitious value $f_{i, j+1}$ and $f_{i-1, j}$ are to be obtained. To consider a more general situation, let $F=\left(f_{i-1, j}, f_{i, j-1}, f_{i+1, j}, f_{i, j+1}\right)^{\mathrm{T}}$. If two secondary fictitious value, $F_{l}$ and $F_{m}$ in vector $F$, can be obtained from Eq. (36). In this particular case, $F_{l}=f_{i+1, j}$ and $F_{m}=f_{i, j-1}$.

$$
\left[\begin{array}{l}
F_{l} \\
F_{m}
\end{array}\right]=\left[\begin{array}{l}
C^{l} \\
C^{m}
\end{array}\right] \cdot U,
$$

then the other two values, $F_{p}$ and $F_{q}$, can be solved by the following procedure. In this particular case, $F_{p}=f_{i, j+1}$ and $F_{q}=f_{i-1, j}$. First, the derivatives $u_{x}^{+}, u_{x}^{-}, u_{y}^{+}$and $u_{y}^{-}$can be represented as follows:

$$
\begin{aligned}
& u_{x}^{+}=\left(w_{1, i-1}, w_{1, i}, w_{1, i+1}\right) \cdot\left(f_{i-1, j}, u_{i, j}, u_{i+1, j}\right)^{\mathrm{T}}, \\
& u_{x}^{-}=\left(w_{1, i-1}, w_{1, i}, w_{1, i+1}\right) \cdot\left(u_{i-1, j}, u_{i, j}-[u], f_{i+1, j}\right)^{\mathrm{T}}, \\
& u_{y}^{+}=\left(w_{1, j-1}, w_{1, j}, w_{1, j+1}\right) \cdot\left(f_{i, j-1}, u_{i, j}, u_{i, j+1}\right)^{\mathrm{T}}, \\
& u_{y}^{-}=\left(w_{1, j-1}, w_{1, j}, w_{1, j+1}\right) \cdot\left(u_{i, j-1}, u_{i, j}-[u], f_{i, j+1}\right)^{\mathrm{T}} .
\end{aligned}
$$

Substituting Eq. (39) into Eqs. (28) and (29), the following equations can be obtained:

$$
A \cdot F=B \cdot U,
$$

where

$$
A=\left[\begin{array}{cccc}
-\sin \theta & \cos \theta & \sin \theta & -\cos \theta \\
\beta^{+} \cos \theta & \beta^{+} \sin \theta & -\beta^{-} \cos \theta & -\beta^{-} \sin \theta
\end{array}\right] \cdot\left[\begin{array}{cccc}
w_{1, i-1} & 0 & 0 & 0 \\
0 & w_{1, j-1} & 0 & 0 \\
0 & 0 & w_{1, i+1} & 0 \\
0 & 0 & 0 & w_{1, j+1}
\end{array}\right]
$$

and

$$
\begin{aligned}
& B=\left[\begin{array}{llllllll}
0 & 0 & 0 & 0 & 0 & 0 & 1 & 0 \\
0 & 0 & 0 & 0 & 0 & 0 & 0 & 1
\end{array}\right] \\
& +\left[\begin{array}{ccccc}
-\sin \theta & \cos \theta & \sin \theta & -\cos \theta \\
\beta^{+} \cos \theta & \beta^{+} \sin \theta & -\beta^{-} \cos \theta & -\beta^{-} \sin \theta
\end{array}\right]\left[\begin{array}{cccccccc}
-w_{1, i} & 0 & 0 & -w_{1, i+1} & 0 & 0 & 0 & 0 \\
-w_{1, j} & 0 & 0 & 0 & -w_{1, j+1} & 0 & 0 & 0 \\
-w_{1, i} & -w_{1, i-1} & 0 & 0 & 0 & w_{1, i} & 0 & 0 \\
-w_{1, j} & 0 & -w_{1, j-1} & 0 & 0 & w_{1, j} & 0 & 0
\end{array}\right] \text {. }
\end{aligned}
$$


Combining Eqs. (38) and (40), the expansions of $F_{p}$ and $F_{q}$ can be easily obtained:

$$
\left[\begin{array}{c}
F_{p} \\
F_{q}
\end{array}\right]=\left[\begin{array}{ll}
A_{1, p} & A_{1, q} \\
A_{2, p} & A_{2, q}
\end{array}\right]^{-1} \cdot\left(B-\left[\begin{array}{ll}
A_{1, l} & A_{1, m} \\
A_{2, l} & A_{2, m}
\end{array}\right] \cdot\left[\begin{array}{c}
C^{l} \\
C^{m}
\end{array}\right]\right) \cdot U .
$$

Now all four primary fictitious values around $(i, j)$ are available. Either Eq. (32) or Eq. (35) can be used as the difference scheme at point $(i, j)$.

\subsubsection{On-interface scheme 3}

Both On-interface schemes 1 and 2 require two off-interface irregular points on the same subdomain. When three out of four surrounding points around on-interface irregular points belong to the same subdomain and one surrounding point is left alone in the other subdomain, On-interface scheme 3 is needed. The situation is illustrated in Fig. 5(a). Points $(i-1, j),(i, j-1),(i, j+1) \in \Omega^{+}$, Point $(i+1, j) \in \Omega^{-}$. To discretize the Poisson equation at point $(i, j)$ in $\Omega^{+}$, the primary fictitious value $f_{i+1, j}$ is required. At point $(i, j), u_{y}^{+}, u_{x}^{-}$and $u_{x}^{+}$can be represented as follows:

$$
\begin{aligned}
& u_{y}^{+}=\left(w_{1, j-1}, w_{1, j}, w_{1, j+1}\right) \cdot\left(u_{i, j-1}, u_{i, j}, u_{i, j+1}\right)^{\mathrm{T}}, \\
& u_{x}^{-}=\left(w_{1, i}^{\prime}, w_{1, i+1}^{\prime}, w_{1, i+2}^{\prime}\right) \cdot\left(u_{i, j}-[u], u_{i+1, j}, u_{i+2, j}\right)^{\mathrm{T}}, \\
& u_{x}^{+}=\left(w_{1, i-1}^{*}, w_{1, i}^{*}, w_{1, i+1}^{*}\right) \cdot\left(u_{i-1, j}, u_{i, j}, f_{i+1, j}\right)^{\mathrm{T}} .
\end{aligned}
$$
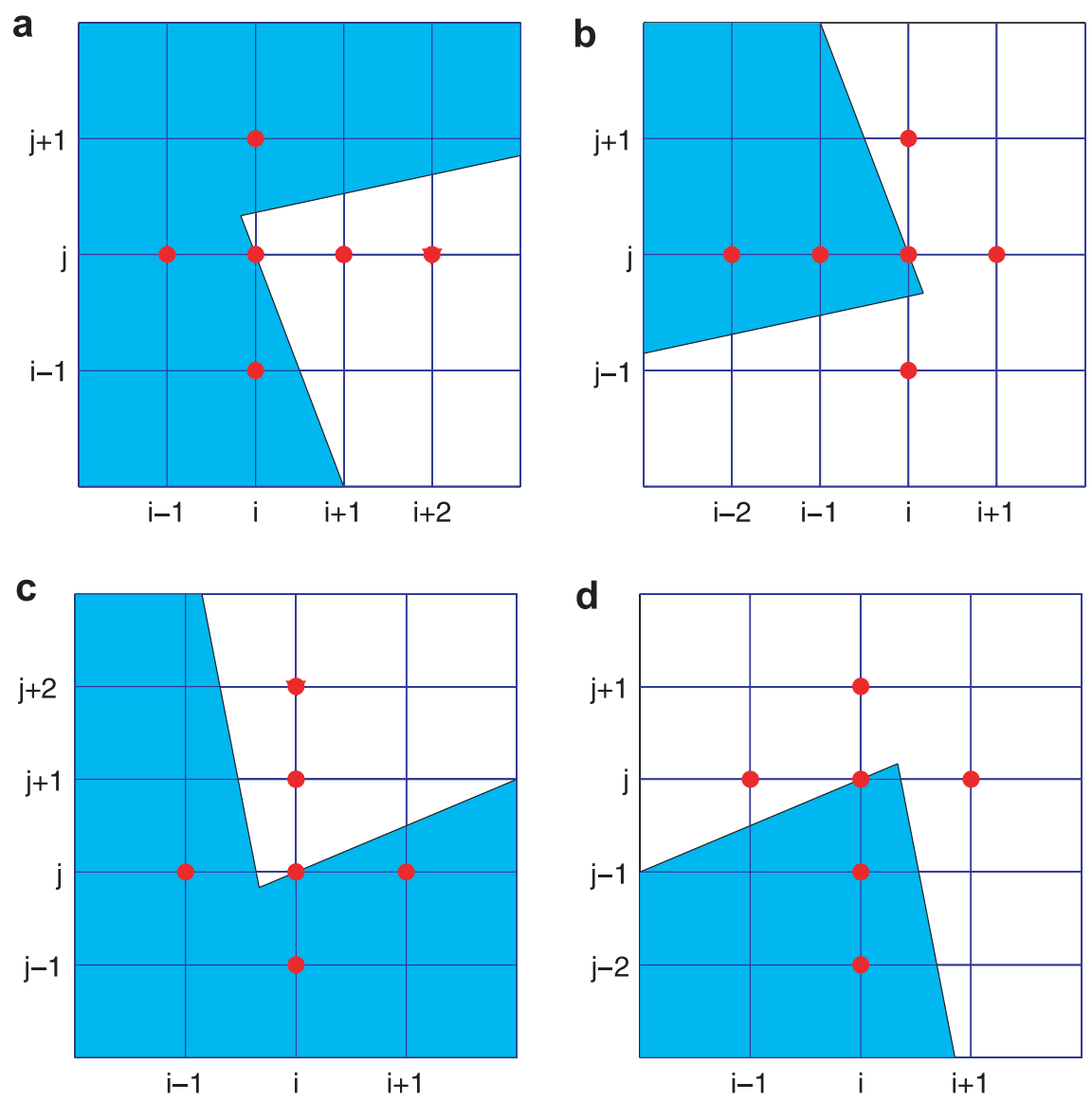

Fig. 5. Four different situations handled by On-interface scheme 3. 
Substituting Eq. (44) into Eq. (7), then solving for $f_{i+1, j}$, the expansion of $f_{i+1, j}$ is obtained as

$$
\begin{aligned}
f_{i+1, j}= & \frac{1}{w_{1, i+1}^{*}}\left(\frac{C_{x}^{-} w_{1, i+2}^{\prime}}{C_{x}^{+}},-w_{1, i-1}^{*},-w_{1, i}^{*}+\frac{C_{x}^{-} w_{1, i}^{\prime}}{C_{x}^{+}}-\frac{C_{y}^{+} w_{1, j}}{C_{x}^{+}}, \frac{C_{x}^{-} w_{1, i+1}^{\prime}}{C_{x}^{+}},-\frac{C_{y}^{+} w_{1, j-1}}{C_{x}^{+}},-\frac{C_{y}^{+} w_{1, j+1}}{C_{x}^{+}},-\frac{C_{x}^{-} w_{1, i}^{\prime}}{C_{x}^{+}},-\frac{\beta^{-} \tan \theta}{C_{x}^{+}}, \frac{1}{C_{x}^{+}}\right) \\
& \cdot\left(u_{i+2, j}, u_{i-1, j}, u_{i, j}, u_{i+1, j}, u_{i, j-1}, u_{i, j+1},[u],\left[u_{\tau}\right],\left[\beta u_{n}\right]\right)^{\mathrm{T}} .
\end{aligned}
$$

The difference scheme of the Poisson equation at point $(i, j)$ can be given in $\Omega^{+}$as Eq. (32).

For the situation in Fig. 5(b), points $(i-1, j),(i, j-1),(i, j+1) \in \Omega^{-}$, point $(i+1, j) \in \Omega^{+}$. The Poisson equation at point $(i, j)$ should be solved in $\Omega^{-}$instead. Therefore, the expansion of $f_{i-1, j}$ in terms of function values is to be determined. Here $u_{y}^{-}, u_{x}^{+}$and $u_{x}^{-}$can be represented as:

$$
\begin{aligned}
& u_{y}^{-}=\left(w_{1, j-1}, w_{1, j}, w_{1, j+1}\right) \cdot\left(u_{i, j-1}, u_{i, j}-[u], u_{i, j+1}\right)^{\mathrm{T}}, \\
& u_{x}^{+}=\left(w_{1, i-2}^{\prime}, w_{1, i-1}^{\prime}, w_{1, i}^{\prime}\right) \cdot\left(u_{i-2, j}, u_{i-1, j}, u_{i, j}\right)^{\mathrm{T}}, \\
& u_{x}^{-}=\left(w_{1, i-1}^{*}, w_{1, i}^{*}, w_{1, i+1}^{*}\right) \cdot\left(f_{i-1, j}, u_{i, j}-[u], u_{i+1, j}\right)^{\mathrm{T}} .
\end{aligned}
$$

Substituting Eq. (44) into Eq. (8), then solving for $f_{i-1, j}$, the expansion of $f_{i-1, j}$ is obtained as

$$
\begin{aligned}
f_{i-1, j}= & \frac{1}{w_{1, i-1}^{*}}\left(\frac{C_{x}^{+} w_{1, i-2}^{\prime}}{C_{x}^{-}}, \frac{C_{x}^{+} w_{1, i-1}^{\prime}}{C_{x}^{-}},-w_{1, i}^{*}+\frac{C_{x}^{+} w_{1, i}^{\prime}}{C_{x}^{-}}-\frac{C_{y}^{-} w_{1, j}}{C_{x}^{-}},-w_{1, i+1}^{*},-\frac{C_{y}^{-} w_{1, j-1}}{C_{x}^{-}},-\frac{C_{y}^{-} w_{1, j+1}}{C_{x}^{-}}, w_{1, i}^{*}+\frac{C_{y}^{-} w_{1, j}}{C_{x}^{-}}, \frac{\beta^{+} \tan \theta}{C_{x}^{-}},-\frac{1}{C_{x}^{-}}\right) \\
& \cdot\left(u_{i-2, j}, u_{i-1, j}, u_{i, j}, u_{i+1, j}, u_{i, j-1}, u_{i, j+1},[u],\left[u_{\tau}\right],\left[\beta u_{n}\right]\right)^{\mathrm{T}} .
\end{aligned}
$$

The difference scheme of the Poisson equation at point $(i, j)$ can be given in $\Omega^{-}$as Eq. (35).

Similarly, for the situation in Fig. 5(c), $u_{y}^{-}, u_{x}^{+}$and $u_{y}^{+}$can be represented as

$$
\begin{aligned}
& u_{y}^{-}=\left(w_{1, j}, w_{1, j+1}, w_{1, j+2}\right) \cdot\left(u_{i, j}-[u], u_{i, j+1}, u_{i, j+2}\right)^{\mathrm{T}}, \\
& u_{x}^{+}=\left(w_{1, i-1}^{\prime}, w_{1, i}^{\prime}, w_{1, i+1}^{\prime}\right) \cdot\left(u_{i-1, j}, u_{i, j}, u_{i+1, j}\right)^{\mathrm{T}}, \\
& u_{y}^{+}=\left(w_{1, j-1}^{*}, w_{1, j}^{*}, w_{1, j+1}^{*}\right) \cdot\left(u_{i, j-1}, u_{i, j}, f_{i, j+1}\right)^{\mathrm{T}} .
\end{aligned}
$$

The expansion of $f_{i, j+1}$ can therefore be obtained by using Eqs. (9) and (48)

$$
\begin{aligned}
f_{i, j+1}= & \frac{1}{w_{1, j+1}^{*}}\left(\frac{C_{y}^{-} w_{1, j+2}}{C_{y}^{+}},-w_{1, j-1}^{*},-w_{1, j}^{*}-\frac{C_{x}^{+} w_{1, i}^{\prime}}{C_{y}^{+}}+\frac{C_{y}^{-} w_{1, j}}{C_{y}^{+}}, \frac{C_{y}^{-} w_{1, j+1}}{C_{y}^{+}},-\frac{C_{x}^{+} w_{1, i-1}^{\prime}}{C_{y}^{+}},-\frac{C_{x}^{+} w_{1, i+1}^{\prime}}{C_{y}^{+}},-\frac{C_{y}^{-} w_{1, j}}{C_{y}^{+}}, \frac{\beta^{-} \cot \theta}{C_{y}^{+}}, \frac{1}{C_{y}^{+}}\right) \\
& \cdot\left(u_{i, j+2}, u_{i, j-1}, u_{i, j}, u_{i, j+1}, u_{i-1, j}, u_{i+1, j},[u],\left[u_{\tau}\right],\left[\beta u_{n}\right]\right)^{\mathrm{T}} .
\end{aligned}
$$

The difference scheme of the Poisson equation at point $(i, j)$ can be given in $\Omega^{+}$as Eq. (32).

For the situation in Fig. 5(d), $u_{y}^{+}, u_{x}^{-}$and $u_{y}^{-}$can be represented as

$$
\begin{aligned}
& u_{y}^{+}=\left(w_{1, j-2}, w_{1, j-1}, w_{1, j}\right) \cdot\left(u_{i, j-2}, u_{i, j-1}, u_{i, j}\right)^{\mathrm{T}}, \\
& u_{x}^{-}=\left(w_{1, i-1}^{\prime}, w_{1, i}^{\prime}, w_{1, i+1}^{\prime}\right) \cdot\left(u_{i-1, j}, u_{i, j}-[u], u_{i+1, j}\right)^{\mathrm{T}}, \\
& u_{y}^{-}=\left(w_{1, j-1}^{*}, w_{1, j}^{*}, w_{1, j+1}^{*}\right) \cdot\left(f_{i, j-1}, u_{i, j}-[u], u_{i, j+1}\right)^{\mathrm{T}} .
\end{aligned}
$$

The expansion of $f_{i, j-1}$ can therefore be obtained by using Eqs. (10) and (50)

$$
\begin{aligned}
f_{i, j-1}= & \frac{1}{w_{1, j-1}^{*}}\left(\frac{C_{y}^{+} w_{1, j-2}}{C_{y}^{-}},-w_{1, j}^{*}+\frac{C_{x}^{-} w_{1, i}^{\prime}}{C_{y}^{-}}+\frac{C_{y}^{+} w_{1, j}}{C_{y}^{-}},-w_{1, j+1}^{*}, \frac{C_{x}^{-} w_{1, i-1}^{\prime}}{C_{y}^{-}}, \frac{C_{x}^{-} w_{1, i+1}^{\prime}}{C_{y}^{-}}, \frac{C_{y}^{+} w_{1, j-1}}{C_{y}^{-}}, w_{1, j}^{*}-\frac{C_{x}^{-} w_{1, i}^{\prime}}{C_{y}^{-}},-\frac{\beta^{+} \cot \theta}{C_{y}^{-}},-\frac{1}{C_{y}^{-}}\right) \\
& \cdot\left(u_{i, j-2}, u_{i, j}, u_{i, j+1}, u_{i-1, j}, u_{i+1, j}, u_{i, j-1},[u],\left[u_{\tau}\right],\left[\beta u_{n}\right]\right)^{\mathrm{T}} .
\end{aligned}
$$

The difference scheme of the Poisson equation at point $(i, j)$ can be given in $\Omega^{-}$as Eq. (35). 
In practice, if not all of three points are on the same side of the interface, secondary fictitious values can be used instead. For example, in Fig. 5(a), if $u_{i, j+1} \in \Omega^{-}$, while the secondary fictitious value $f_{i, j+1}$ is already resolved, Eq. (45) can still be used for the primary fictitious value $f_{i+1, j}$ by replacing $u_{i, j+1}$ with the secondary fictitious value $f_{i, j+1}$.

\subsection{A pseudo-code of $M I B$}

1. If $((i, j)$ is a regular point), call standard five-point FD scheme

2. If $\left((i, j)\right.$ is an irregular point and $\left.(i, j) \in \Omega^{+}\right)$then

- If $\left((i+1, j) \in \Omega^{-}\right)$then

- If (both auxiliary points are in 'proper' region and enough points for $u_{y}^{+}$or $u_{y}^{-}$) then

* call Off-interface scheme 1

- else store the unsolved point $(i, j)$

- If any other surrounding point is in $\Omega^{-}$, a similar procedure is applied.

3. If $((i, j) \in \Gamma)$ then

- If (exact two surrounding points stay on the same side of the interface)

- If (two auxiliary points on $x$ and $y$ directions are on the same side of the interface) then

* call On-interface scheme 1

- elseif (two auxiliary points are on the different side of the interface) then

* call On-interface scheme 2

- else (cannot find two auxiliary points)

* store the unsolved point $(i, j)$

- else store the unsolved point $(i, j)$

4. Revisit stored unsolved point $(i, j)$, use secondary fictitious values

- If $\left((i, j) \in \Omega^{+}\right)$then

- If $\left((i+1, j) \in \Omega^{-}\right)$then

* determine $n$, the number of unknowns, after introducing secondary fictitious values among $F_{i, j}, F_{i+1, j}, F_{i-1, j}$ and $F_{i+2, j}$

* If $(n==0)$ then

- all surrounding points are real unknowns or have primary fictitious value now, call standard five-point FD scheme

* If $(n==1)$ then

- call Off-interface scheme 2(b);

$*$ elseif $(n==2)$ then

- call Off-interface scheme 2(a);

* else

- pause

If (any other surrounding points are in $\Omega^{-}$), similar procedure is applied.

- If $((i, j) \in \Gamma)$ then

- If (all surrounding points are real unknowns or have primary fictitious value now), call the standard five-point FD scheme.

- If (three surrounding points are on the same side or have secondary fictitious value on the same side), then

* call On-interface scheme 3

- else pause

- If $\left((i, j)\right.$ is an irregular point and $\left.(i, j) \in \Omega^{-}\right)$, use primary fictitious values and call the standard five-point FD scheme.

\subsection{The critical value of the acute angle}

The standard MIB method can always handle a $C^{1}$ irregular interface even if it has large curvature, since enough auxiliary points can always be found by refining mesh. When the interface is Lipschitz continuous but 
not $C^{1}$, refining mesh usually cannot provide required points around a kink. The present generalized MIB method overcomes the difficulty by using secondary fictitious values to substitute the function of unavailable auxiliary points.

Fig. 6(a) shows the smallest acute angle that the generalized MIB can handle when a vertical or horizontal line bisects the angle. The number 1, 2, 3 labels the sequence of the fictitious values that were resolved. The fictitious values at points labeled 1 were found with On-interface scheme 1 . Then the fictitious values at points labeled 2 were found by using points labeled 1 with On-interface scheme 3 . Finally the fictitious values at points labeled 3 were found by using secondary fictitious values at points labeled 1 and 2 with Off-interface scheme 3. If the angle is smaller than this one, the fictitious values at $(i-1, j+2)$ and $(i+1, j+2)$ cannot be founded, and the fictitious value at $(i, j+2)$ is also not available. Therefore, $2 \tan ^{-1}\left(\frac{1}{3}\right)$ is the critical value of the smallest angle that the present MIB can handle with 2nd order accuracy. Any angle that is larger than the critical value can always be treated with the designed 2 nd order accuracy.

In most cases, the mesh line that bisects the angle is not vertical or horizontal. Fig. 6(b) shows a situation in which the angle is smaller than the critical value and it can still be handled by the MIB. The fictitious values at points labeled 1 were found with On-interface scheme 1 and Off-interface scheme 1 . Then the fictitious values at points labeled 2 were found with On-interface scheme 3 . Finally the fictitious values at points labeled 3 were found by using secondary fictitious values at points labeled 1 and 2 with Off-interface scheme 3. However if the angle is very small such that $(i-1, j+3)$ and $(i, j+3)$ are both in $\Omega^{-}$, the present MIB method will not be able to capture the shape of the corner. In the case of Fig. 6, the present method has 2 nd order accuracy if and only if the third horizontal mesh line above the tip contains at least two points inside the acute angle.

\section{Numerical studies}

In this section, we examine the performance of the proposed MIB scheme for solving the Poisson equation with sharp-edged interfaces, thin-layered interfaces and interfaces that intersect with the geometric boundary. We consider six different interface geometries coupled with various boundary conditions and solution behaviors. In the first test case, we examine capability of the present MIB method in treating the critical sharp edge. We also test the present scheme for grid points exactly at the tip of the sharp edge, for which the interface jump conditions are not unique due to undefined derivatives. The second case is designed to test the present method for handling multiple sharp edges and oscillatory solutions. Case 3 is a missile geometry. Case 4 has multiply connected domains. In Case 5, the level set function is not piecewise linear. In Case 6, we consider a thin-layered interface geometry. Finally, we demonstrate the proposed method with a problem having weak
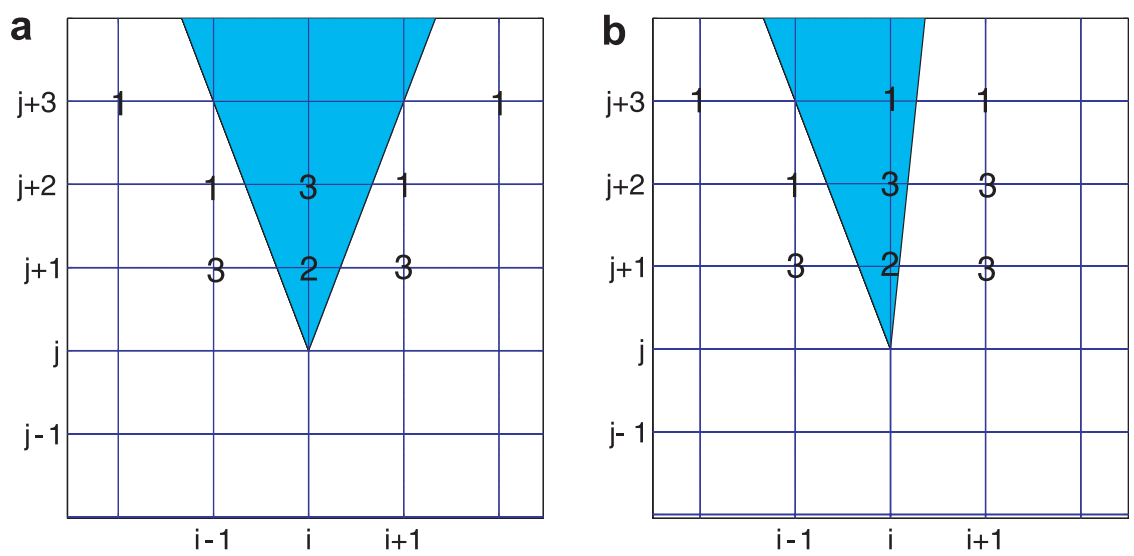

Fig. 6. (a) The critical value of the smallest angle that MIB can handle; (b) an angle smaller than the critical value. 
solution. In all the cases, numerical results are compared to analytical ones. The standard $L_{\infty}$ norm error measurement is employed in this section.

\section{Case 1}

We consider the 2D Poisson equation

$$
\left(\beta u_{x}\right)_{x}+\left(\beta u_{y}\right)_{y}=q(x, y)
$$

defined in a square domain $[-1,1] \times[-1,1]$. The exact solution is designed to be

$$
u^{+}(x, y)=x^{2}+y^{2}+1, \quad u^{-}(x, y)=\sin (\pi x) \sin (\pi y),
$$

with piecewise continuous coefficients

$$
\beta^{+}(x, y)=\left(x^{2}-y^{2}+3\right) / 7, \quad \beta^{-}(x, y)=(x y+2) / 5 .
$$

As shown in Figs. 7 and 8, interfaces with both obtuse and acute angles are considered. Level-set functions $\phi$ of the interfaces are given as:

\section{- Case 1(a)}

$$
\phi(x, y)= \begin{cases}y+x / 2 & x<0 \\ y-2 x & x \geqslant 0 .\end{cases}
$$

- Case 1(b)

$$
\phi(x, y)= \begin{cases}y+3 x & x<0 \\ y-3 x & x \geqslant 0 .\end{cases}
$$

- Case 1(c)

$$
\phi(x, y)= \begin{cases}y+3 x & x<0 \\ y-11 x & x \geqslant 0 .\end{cases}
$$

Numerical results are presented in Table 1. Second order convergence is observed in all the three cases.

As the derivative at the tip of the sharp edge is undefined, it is interesting to examine the performance of the present method for treating irregular points 'on the tip'. To this end, we place the tip on a grid point during the mesh refinement and computer the error rate. Since the jump condition involves the derivative on the interface, we need to compute derivatives at the tip if it is on a grid point. As shown in Fig. 8, the derivative at the tip of the sharp edge can be computed as asymptotic limits from either the left edge or the right edge shifting toward the tip. These limits are not equal, and leads to two flux jump conditions. We need using only one of these flux jump conditions. An interesting question is what happens to the solution if different flux jump conditions are used. Our numerical test is based on the interface geometry of Case 1(c). We modify the solution from $u^{-}(x, y)=\sin (\pi x) \sin (\pi y)$ to $u^{-}(x, y)=\cos (\pi x) \sin (\pi y)$ to avoid zero at $x=0$. The errors computed by using two flux jump conditions are presented in Table 2. It is seen that the errors in two computations are both of second order, and differ very little. A further error comparison is conducted at the tip point $(0,0.1)$ using a $20 \times 20$ mesh. The solution error from using the left flux jump condition is $1.02845419680633 \times 10^{-2}$ and that from using the right flux jump condition is $1.02845419680637 \times 10^{-2}$. Similar behavior is found in other examples. Therefore, we conclude that the proposed MIB method works well for grid points at the tip of sharp edges.

\section{Case 2}

In this case, we test the presented method with a more complicated geometry. As shown in Fig. 9, the levelset function $\phi$ of a pentagon star is given as: 

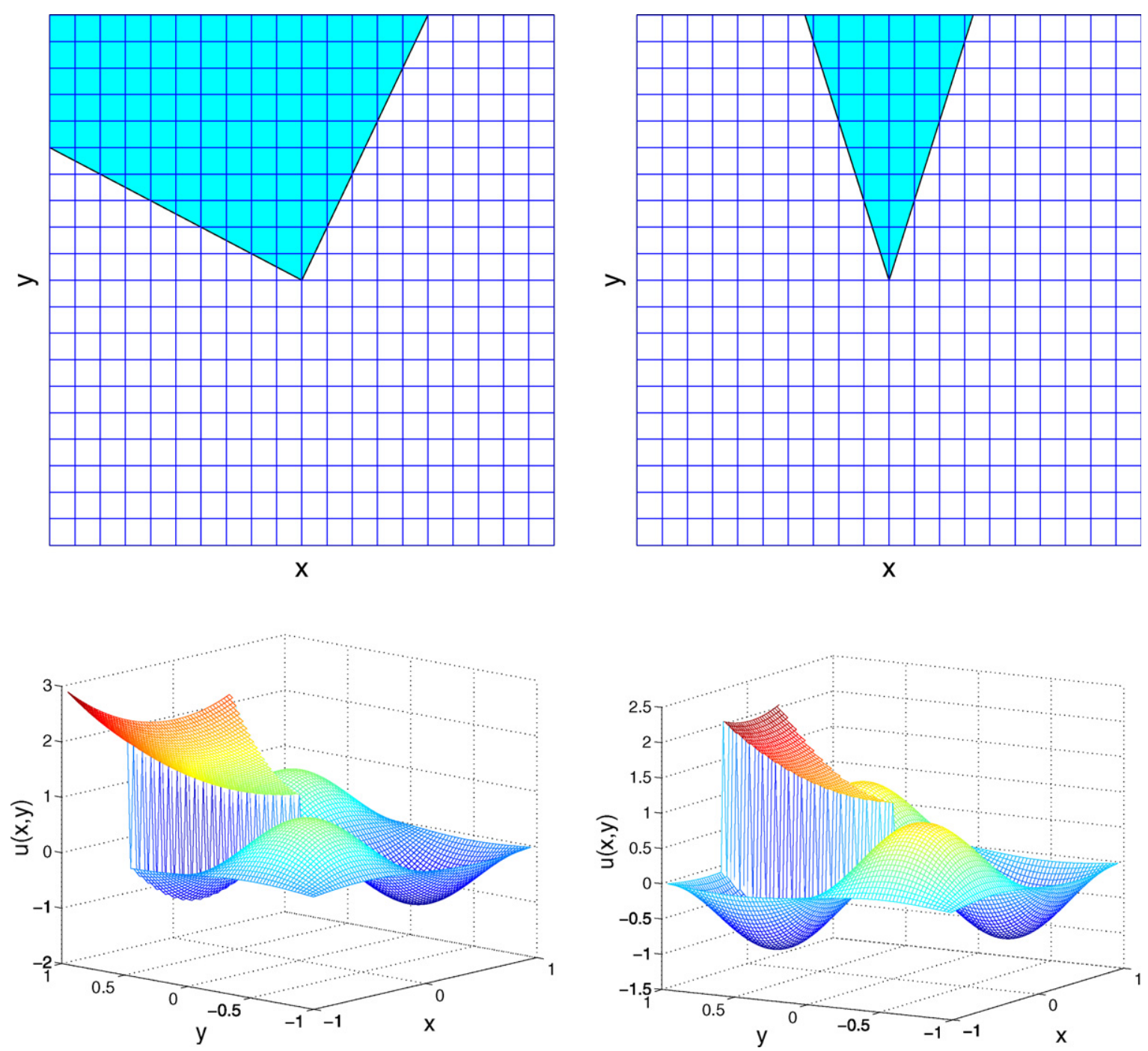

Fig. 7. Interfaces on $20 \times 20$ meshes (top row) and the computed solution (bottom row) for the 2D Poisson equation (Cases 1 (a) and 1(b))

$$
\phi(r, \theta)= \begin{cases}\frac{R \sin \left(\theta_{t} / 2\right)}{\sin \left(\theta_{t} / 2+\theta-\theta_{r}-2 \pi(i-1) / 5\right)}-r & \theta_{r}+\pi(2 i-2) / 5 \leqslant \theta<\theta_{r}+\pi(2 i-1) / 5, \\ \frac{R \sin \left(\theta_{t} / 2\right)}{\sin \left(\theta_{t} / 2-\theta+\theta_{r}+2 \pi(i-1) / 5\right)}-r & \theta_{r}+\pi(2 i-3) / 5 \leqslant \theta<\theta_{r}+\pi(2 i-2) / 5,\end{cases}
$$

with $\theta_{t}=\frac{\pi}{5}, \theta_{r}=\frac{\pi}{7}, R=\frac{6}{7}$ and $i=1,2,3,4,5$. The discontinuous coefficients are given by

$$
\beta^{+}(x, y)=1, \quad \beta^{-}(x, y)=2+\sin (x+y) .
$$

Four different solutions are designed with variable amount of oscillations:

\section{- Case 2(a)}

$$
u^{+}(x, y)=8, \quad u^{-}(x, y)=x^{2}+y^{2}+\sin (x+y) .
$$

- Case 2(b)

$$
u^{+}(x, y)=5+5\left(x^{2}+y^{2}\right), \quad u^{-}(x, y)=x^{2}+y^{2}+\sin (x+y) .
$$



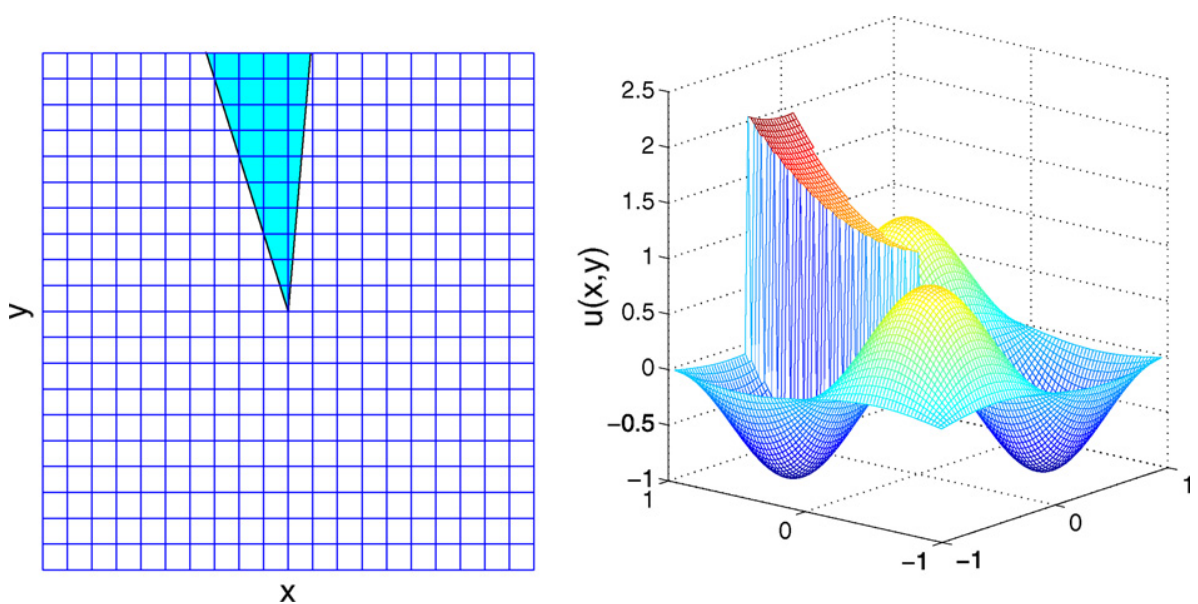

Fig. 8. Interface on a $20 \times 20$ mesh (left) and the computed solution (right) for the $2 \mathrm{D}$ Poisson equation (Case 1(c)).

Table 1

Numerical efficiency tests of the 2D Poisson equation (Case 1)

\begin{tabular}{|c|c|c|c|c|c|c|}
\hline \multirow[t]{2}{*}{$n_{x} \times n_{y}$} & \multicolumn{2}{|l|}{ Case 1(a) } & \multicolumn{2}{|c|}{ Case 1(b) } & \multicolumn{2}{|l|}{ Case 1(c) } \\
\hline & $L_{\infty}$ & Order & $L_{\infty}$ & Order & $L_{\infty}$ & Order \\
\hline $20 \times 20$ & $1.02 \mathrm{e}-2$ & & $9.12 \mathrm{e}-3$ & & $9.75 \mathrm{e}-3$ & \\
\hline $40 \times 40$ & $2.82 \mathrm{e}-3$ & 1.85 & $2.22 \mathrm{e}-3$ & 2.03 & $2.39 \mathrm{e}-3$ & 2.03 \\
\hline $80 \times 80$ & $7.33 e-4$ & 1.94 & $5.64 \mathrm{e}-4$ & 1.98 & $5.89 \mathrm{e}-4$ & 2.02 \\
\hline $160 \times 160$ & $1.86 \mathrm{e}-4$ & 1.98 & $1.42 \mathrm{e}-4$ & 1.99 & $1.48 \mathrm{e}-4$ & 1.99 \\
\hline
\end{tabular}

Table 2

Comparison of numerical solutions involving the left and right flux jump conditions at the tip in the geometry of Case 1(c)

\begin{tabular}{|c|c|c|c|c|}
\hline \multirow[t]{2}{*}{$n_{x} \times n_{y}$} & \multicolumn{2}{|c|}{ Left flux jump condition } & \multicolumn{2}{|c|}{ Right flux jump condition } \\
\hline & $L_{\infty}$ & Order & $L_{\infty}$ & Order \\
\hline $20 \times 20$ & $1.16 \mathrm{e}-2$ & & $1.15 \mathrm{e}-3$ & \\
\hline $40 \times 40$ & $2.67 \mathrm{e}-3$ & 2.11 & $2.66 \mathrm{e}-3$ & 2.11 \\
\hline $80 \times 80$ & $6.69 \mathrm{e}-4$ & 2.00 & $6.68 \mathrm{e}-4$ & 2.00 \\
\hline $160 \times 160$ & $1.68 \mathrm{e}-4$ & 2.00 & $1.69 \mathrm{e}-4$ & 1.98 \\
\hline
\end{tabular}

\section{- Case 2(c)}

$$
u^{+}(x, y)=6+\sin (2 \pi x) \sin (2 \pi y), \quad u^{-}(x, y)=x^{2}+y^{2}+\sin (x+y) .
$$

\section{- Case 2(d)}

$$
u^{+}(x, y)=6+\sin (6 \pi x) \sin (6 \pi y), \quad u^{-}(x, y)=x^{2}+y^{2}+\sin (x+y) .
$$

In each case, the jumps in $u$ and $u_{n}$ along the interface can be evaluated from the solution. Table 3 gives the numerical results of the second order MIB method for this difficult problem. These results are also illustrated in Fig. 10. Obviously, Case 2(d) is a very challenging numerical example. It consists of three difficulties, i.e., material interface, sharp edge and oscillatory solution. Similar problems described by the Helmholtz equation has much impact to computational electromagnetics. 


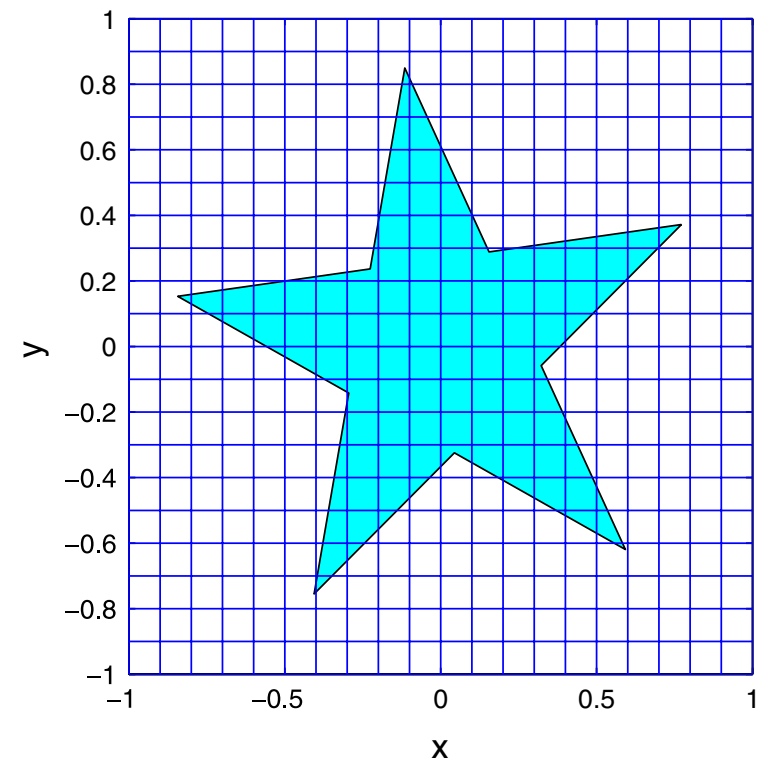

Fig. 9. The pentagon star interface in a $20 \times 20$ mesh (Case 2).

Table 3

Numerical efficiency tests of the pentagon star interface (Case 2(a)-(d))

\begin{tabular}{|c|c|c|c|c|c|c|c|c|}
\hline \multirow[t]{2}{*}{$n_{x} \times n_{y}$} & \multicolumn{2}{|l|}{ Case 2(a) } & \multicolumn{2}{|c|}{ Case 2(b) } & \multicolumn{2}{|l|}{ Case 2(c) } & \multicolumn{2}{|c|}{ Case 2(d) } \\
\hline & $L_{\infty}$ & Order & $L_{\infty}$ & Order & $L_{\infty}$ & Order & $L_{\infty}$ & Order \\
\hline $20 \times 20$ & $6.11 \mathrm{e}-4$ & & $6.11 \mathrm{e}-4$ & & $5.26 \mathrm{e}-2$ & & $9.72 \mathrm{e}-1$ & \\
\hline $40 \times 40$ & $6.07 \mathrm{e}-5$ & 3.33 & $6.07 \mathrm{e}-5$ & 3.33 & $8.51 \mathrm{e}-3$ & 2.62 & $1.94 \mathrm{e}-1$ & 2.32 \\
\hline $80 \times 80$ & $1.34 \mathrm{e}-5$ & 2.18 & $1.34 \mathrm{e}-5$ & 2.18 & $2.39 \mathrm{e}-3$ & 1.83 & $5.49 \mathrm{e}-2$ & 1.82 \\
\hline $160 \times 160$ & $4.15 \mathrm{e}-6$ & 1.69 & $4.15 e-6$ & 1.69 & $6.64 \mathrm{e}-4$ & 1.85 & $1.48 \mathrm{e}-2$ & 1.89 \\
\hline
\end{tabular}

We also discuss the following two cases with the same geometries but with homogeneous source terms (i.e., $q(x, y)=0$ in Eq. (52)). We set $\beta \equiv 1$.

\section{- Case 2(e)}

$$
u^{+}(x, y)=0, \quad u^{-}(x, y)=\exp (x) \sin (y) .
$$

\section{- Case 2(f)}

$$
u^{+}(x, y)=0, \quad u^{-}(x, y)=\exp (\pi x) \sin (\pi y) .
$$

The numerical errors of the homogeneous cases are listed in Table 4. The numerical solutions of the homogeneous cases are also illustrated in Fig. 10. Comparing the computed solutions in Cases 2(a)-(d) to those in Case 2(e)-(f), there is no significant difference between homogeneous cases and non-homogeneous cases. The accuracy of the computed solutions strongly depend on the natural of the solution itself. The solutions that vary rapidly with respect to spatial coordinates are more difficult to capture than those that vary slowly. Therefore, Cases 2(d) and 2(f) have relatively larger errors than others. However, it can be seen that all the above cases have achieved second order convergence. The convergence is not quite uniform because the relative location of tips with respect to mesh lines varies as the grid is refined.

\section{Case 3}

Here we solve the Poisson equation for a missile geometry. The geometric parameters is given in Fig. 11. In the present computation, the missile is rotated $60^{\circ}$ counterclockwise. All the parameters but the angular ones 
a
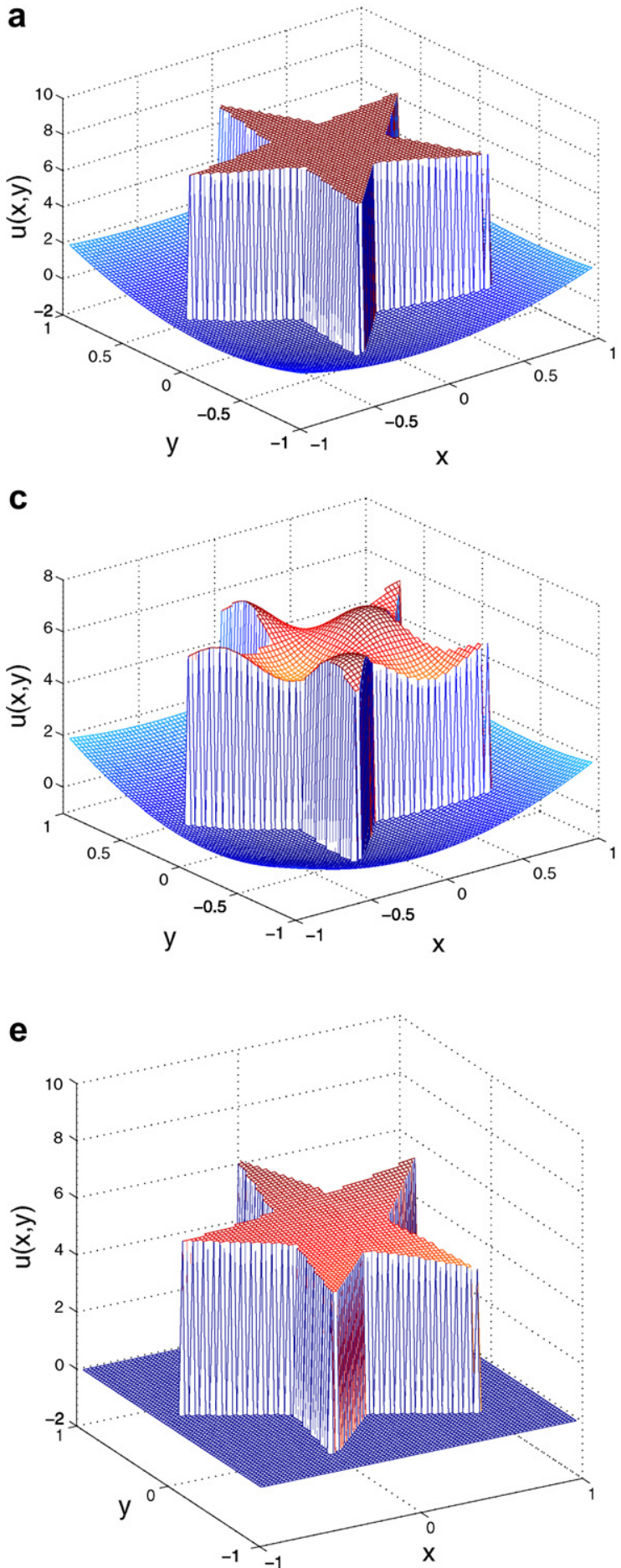

b

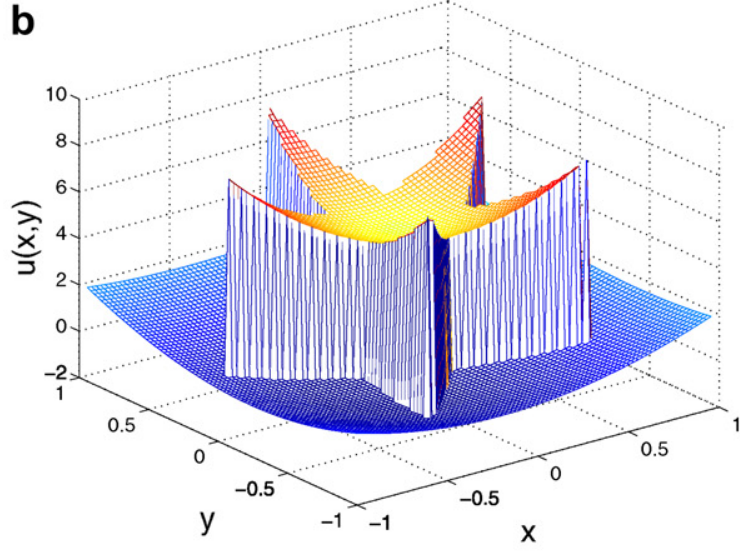

d

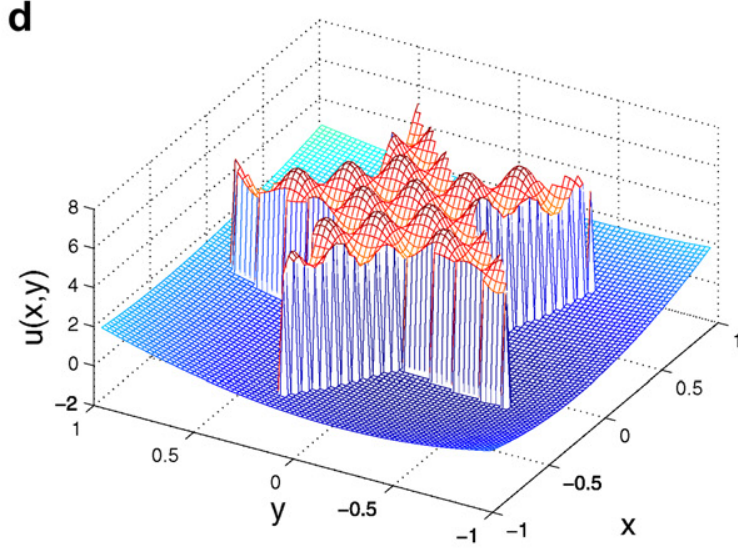

f

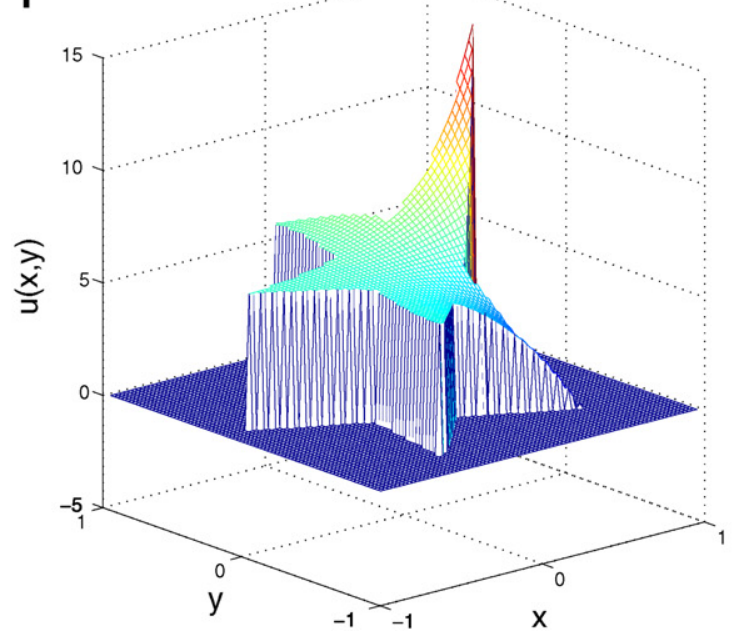

Fig. 10. Computed solution for the pentagon star interface (Cases 2(a), 2(b), 2(c), 2(d), 2(e) and 2(f)).

are divided by 100 to fit in $[-1,1] \times[-1,1]$ computational domain. Fig. 12(a) shows the missile interface on a $20 \times 20$ mesh. The coordinate of center $C$, which is labeled in Fig. 11 , is $(-0.4,-0.4)$. In this case, the exact solution is given by: 
Table 4

Numerical efficiency tests of the pentagon star interface (Cases 2(e)-2(f))

\begin{tabular}{|c|c|c|c|c|}
\hline \multirow[t]{2}{*}{$n_{x} \times n_{y}$} & \multicolumn{2}{|l|}{ Case 2(e) } & \multicolumn{2}{|l|}{ Case 2(f) } \\
\hline & $L_{\infty}$ & Order & $L_{\infty}$ & Order \\
\hline $20 \times 20$ & $3.84 \mathrm{e}-4$ & & $6.74 \mathrm{e}-2$ & \\
\hline $40 \times 40$ & $7.34 \mathrm{e}-5$ & 2.39 & $1.02 \mathrm{e}-2$ & 2.72 \\
\hline $80 \times 80$ & $1.94 \mathrm{e}-5$ & 1.92 & $4.60 \mathrm{e}-3$ & 1.15 \\
\hline $160 \times 160$ & $3.23 \mathrm{e}-6$ & 2.59 & $7.43 \mathrm{e}-4$ & 2.63 \\
\hline
\end{tabular}

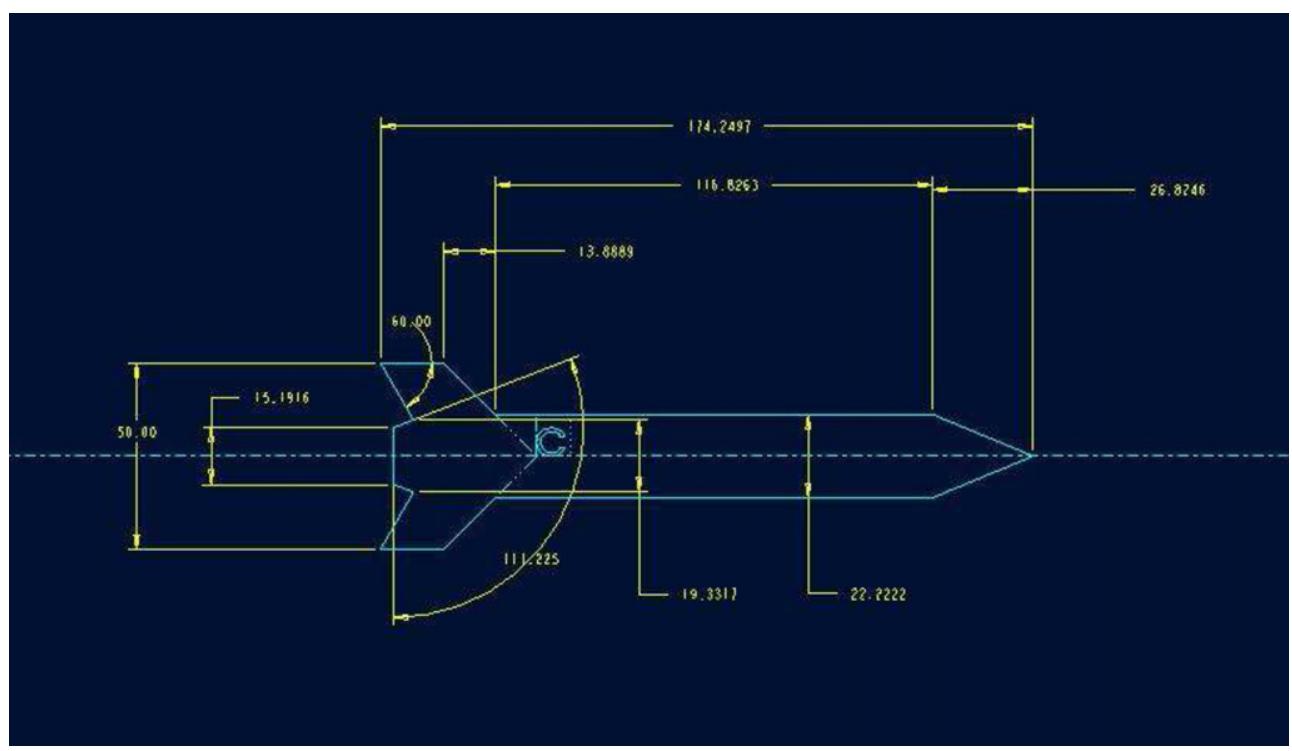

Fig. 11. The configuration of a missile (Case 3).
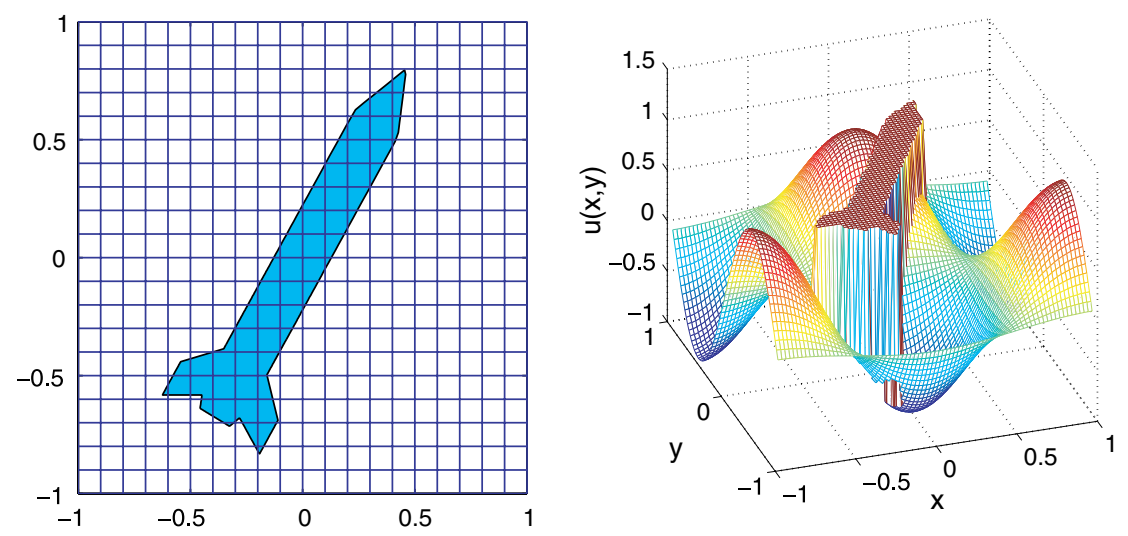

Fig. 12. The missile interface on a $20 \times 20$ mesh (left) and computed solution (right) (Case 3).

$$
u^{+}(x, y)=1, \quad u^{-}(x, y)=\cos (\pi x) \sin (\pi y),
$$

with discontinuous coefficients

$$
\beta^{+}(x, y)=\frac{x^{2}-y^{2}+3}{7}, \quad \beta^{-}(x, y)=\frac{x y+2}{5} .
$$

Numerical results in Table 5 verify the convergence of the proposed method. 


\section{Case 4}

Here we solve the Poisson equation on a piece of twisted chess board, see Fig. 13. The level set function $\phi$ of the interface is given as

$$
\phi(x, y)=-\left(y-x-\frac{1}{3}\right)\left(y-x+\frac{1}{3}\right)\left(y+\frac{x}{3}-\frac{1}{2}\right)\left(y+\frac{x}{3}+\frac{1}{2}\right) .
$$

The discontinuous coefficients are given by

$$
\beta^{+}(x, y)=1, \quad \beta^{-}(x, y)=2+\sin (x+y) .
$$

Two different solutions are designed.

\section{- Case 4(a)}

$$
u^{+}(x, y)=8, \quad u^{-}(x, y)=x^{2}+y^{2}+\sin (x+y) .
$$

\section{- Case 4(b)}

$$
u^{+}(x, y)=8+3 x y, \quad u^{-}(x, y)=x^{2}+y^{2}+\sin (x+y) .
$$

In this case, there are several irregular points near the domain boundary whose primary fictitious values cannot be solved by interface scheme because the auxiliary points of the irregular points on the boundary are not available. As shown in $20 \times 20$ mesh in Fig. 13, the primary fictitious value at $(1.0,0.6)$ of point

Table 5

Numerical accuracy tests of the missile interface case (Case 3)

\begin{tabular}{lll}
\hline$n_{x} \times n_{y}$ & $L_{\infty}$ & Order \\
\hline $20 \times 20$ & $5.68 \mathrm{e}-3$ & 1.73 \\
$40 \times 40$ & $1.71 \mathrm{e}-3$ & 1.98 \\
$80 \times 80$ & $4.33 \mathrm{e}-4$ & 1.99 \\
$160 \times 160$ & $1.09 \mathrm{e}-4$ & \\
\hline
\end{tabular}

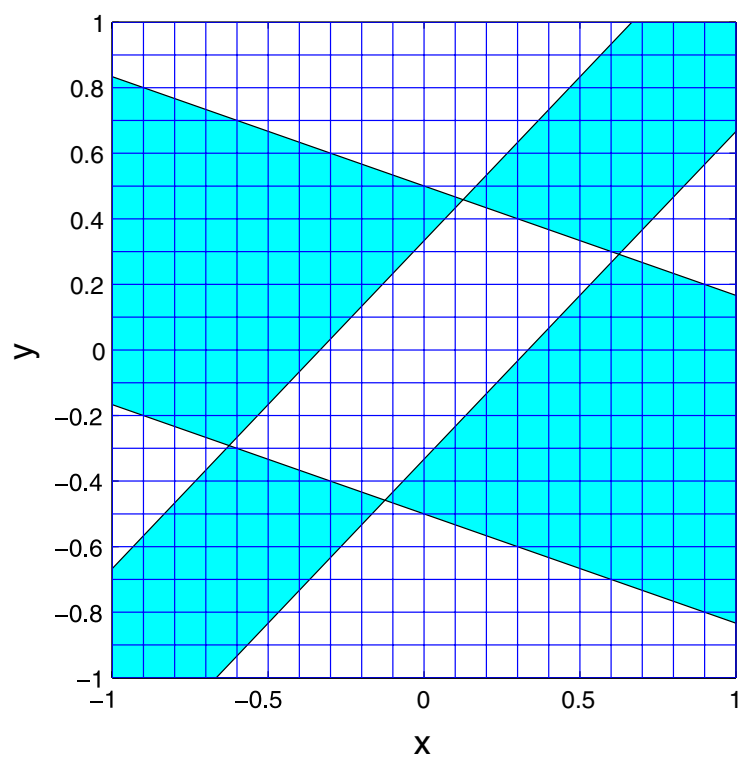

Fig. 13. The chess board interface on a $20 \times 20$ mesh (Case 4). 
$(0.9,0.6)$ cannot be determined by the interface scheme mentioned before. Under Dirichlet boundary condition, we could discretize $\left(\beta u_{x}\right)_{x}$ at $(0.9,0.6)$ by using a one-sided finite difference scheme, which involves three points $(0.7,0.6),(0.8,0.6)$ and $(0.9,0.6)$. Other boundary conditions will not be discussed in the present paper.

Numerical results in Table 6 verify the convergence of the proposed method and numerical solution of Cases 4(a) and 4(b) are shown in Fig. 14.

\section{Case 5}

In this example, we considered a problem with multiply connected curved edges, see Fig. 15. The level set function $\phi(x, y)$ is given by

$$
\phi(x, y)=-\left(y-4 x^{2}+\frac{1}{4}\right)\left(y+4 x^{2}-\frac{1}{3}\right) .
$$

In this case, the interface $\Gamma$ is consist of six pieces of smooth curves. The exact solution is given by:

$$
u^{+}(x, y)=8+3 x y, \quad u^{-}(x, y)=x^{2}+y^{2}+\sin (x+y),
$$

with discontinuous coefficients

$$
\beta^{+}(x, y)=1, \quad \beta^{-}(x, y)=2+\sin (x+y) .
$$

Numerical results are presented in Table 7. Second order convergence is achieved.

\section{Case 6}

We consider a thin-layered interface problem which is important to evaluating thin-layered coatings, see Fig. 16. In this case, the whole domain is divided into three subdomains, thin layer $\Omega^{+}$, outside domain $\Omega_{0}^{-}$ and inside domain $\Omega_{i}^{-}$. The interface between $\Omega_{0}^{-}$and $\Omega^{+}$is $\Gamma_{0}$ and the interface between $\Omega_{i}^{-}$and $\Omega^{+}$is $\Gamma_{i}$. There are two types of irregular points. Type I irregular points are near $\Gamma_{0}$ and their five-point FD scheme involves the points from both $\Omega^{+}$and $\Omega_{0}^{-}$. Type II irregular points are near $\Gamma_{i}$ and their five-point FD scheme involves the points from both $\Omega^{+}$and $\Omega_{i}^{-}$. Both types irregular points can be solved by the present MIB
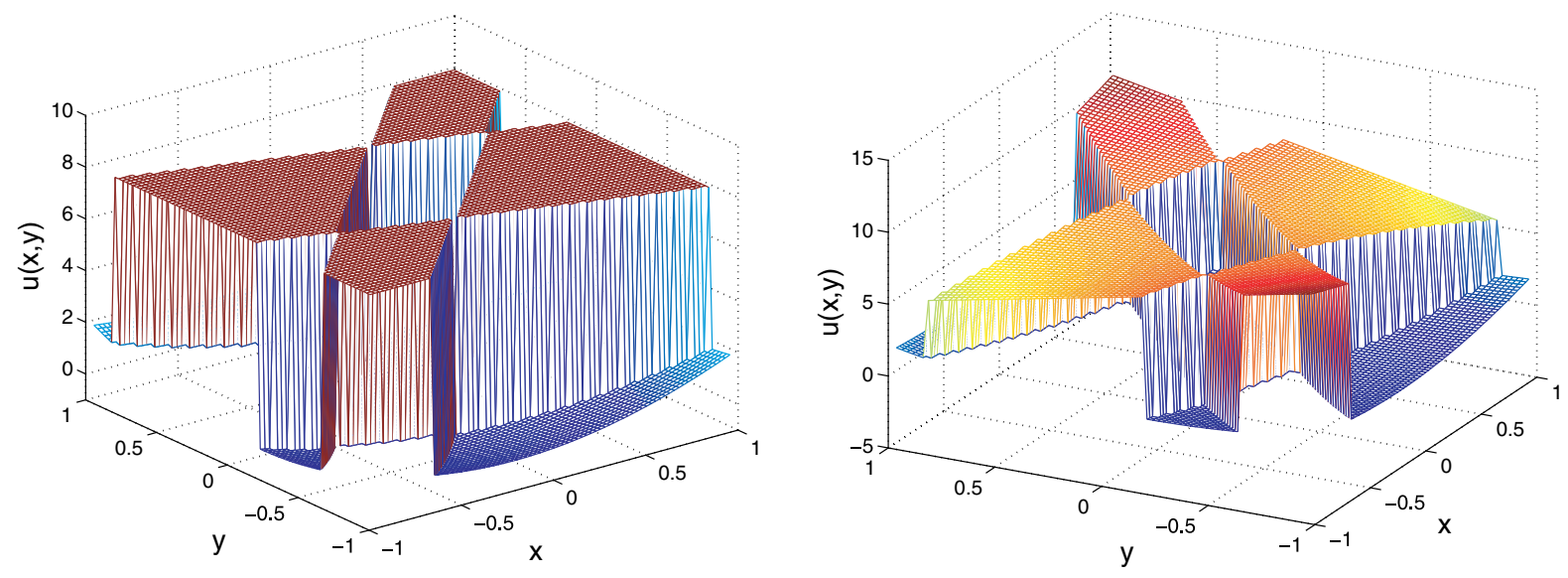

Fig. 14. Computed solutions for the chess board interface (Cases 4(a) and 4(b))

\begin{tabular}{|c|c|c|c|c|}
\hline \multirow[t]{2}{*}{$n_{x} \times n_{y}$} & \multicolumn{2}{|l|}{ Case 4(a) } & \multicolumn{2}{|l|}{ Case 4(b) } \\
\hline & $L_{\infty}$ & Order & $L_{\infty}$ & Order \\
\hline $20 \times 20$ & $5.92 \mathrm{e}-4$ & & $5.97 \mathrm{e}-4$ & \\
\hline $40 \times 40$ & $1.50 \mathrm{e}-4$ & 1.98 & $1.50 \mathrm{e}-4$ & 1.99 \\
\hline $80 \times 80$ & $4.26 \mathrm{e}-5$ & 1.82 & $4.26 \mathrm{e}-5$ & 1.82 \\
\hline $160 \times 160$ & $9.40 \mathrm{e}-6$ & 2.18 & $9.40 \mathrm{e}-6$ & 2.18 \\
\hline
\end{tabular}

Table 6

Numerical accuracy tests of the chess board interface case (Cases 4(a) and 4(b)) 

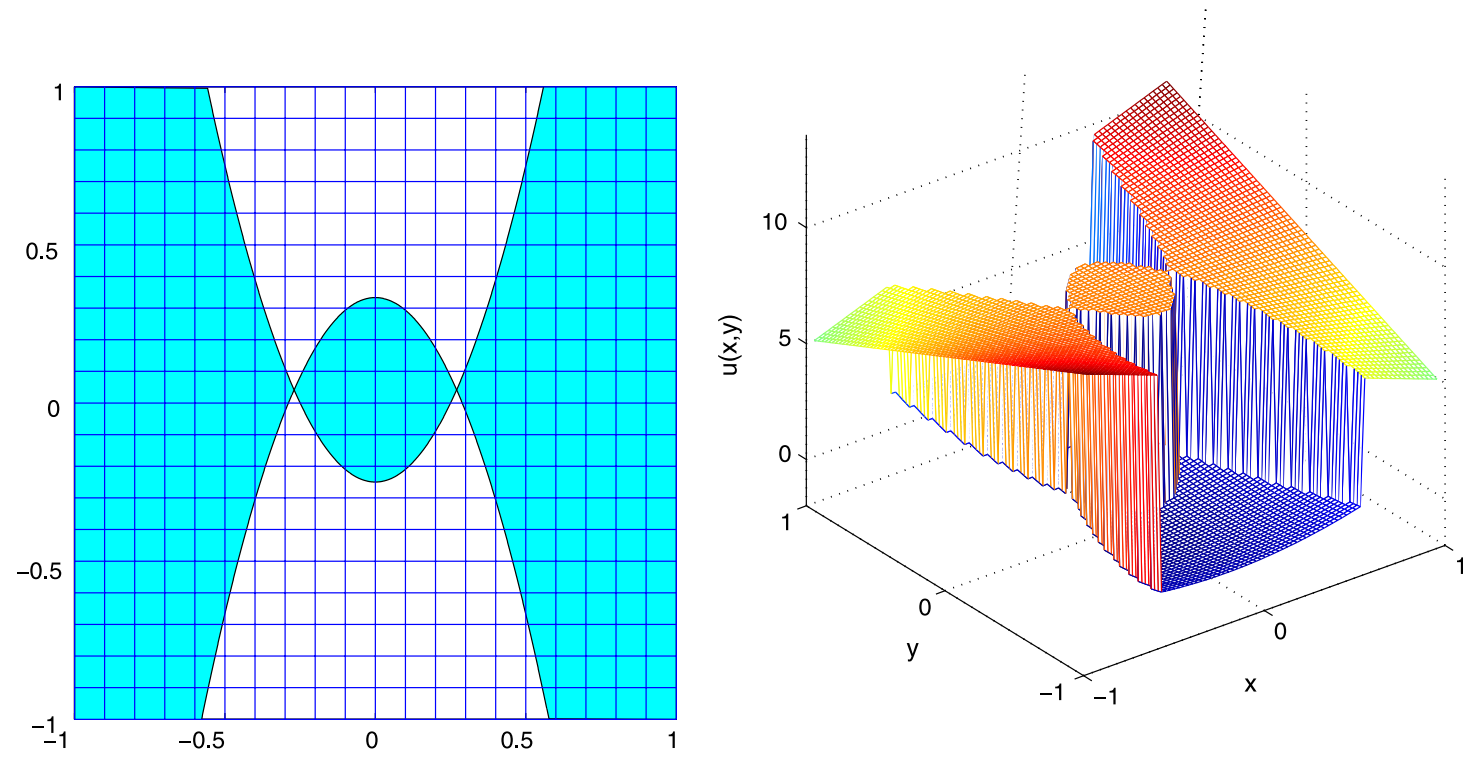

Fig. 15. The interface of Case 5 on a $20 \times 20$ mesh (left) and computed solution on a $80 \times 80$ mesh (right).

Table 7

Numerical accuracy tests of Case 5

\begin{tabular}{llr}
\hline$n_{x} \times n_{y}$ & $L_{\infty}$ & Order \\
\hline $20 \times 20$ & $6.71 \mathrm{e}-4$ & \\
$40 \times 40$ & $1.71 \mathrm{e}-4$ & 1.97 \\
$80 \times 80$ & $5.71 \mathrm{e}-5$ & 1.58 \\
$160 \times 160$ & $8.32 \mathrm{e}-6$ & 2.78 \\
\hline
\end{tabular}
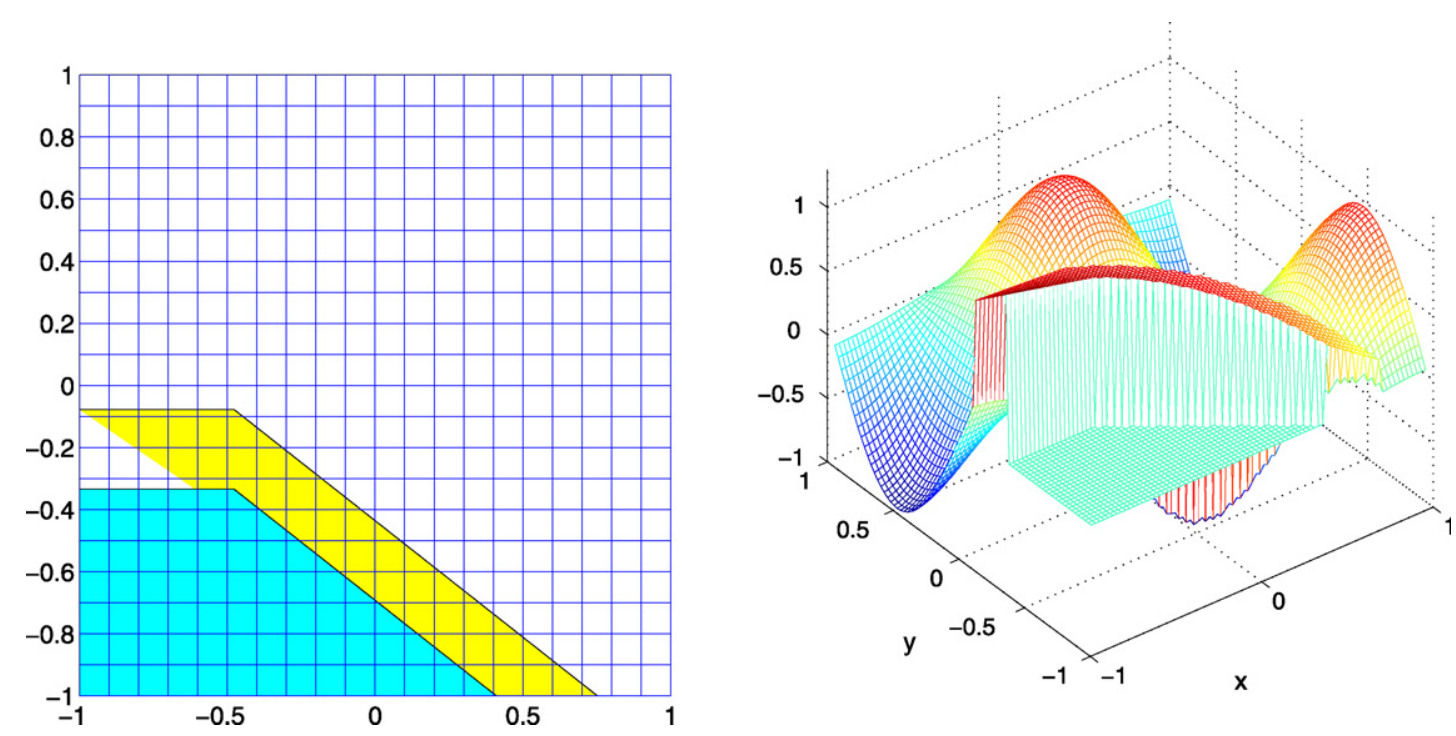

Fig. 16. The thin layer interface on a $20 \times 20$ mesh (left) and computed solution (right) (Case 6).

method directly. However, when we consider the practice problems, the material within $\Omega_{i}^{-}$is usually perfect conductor, therefore we have $u(x, y) \equiv 0$ within $\Omega_{i}^{-}$. In this case, the scheme for Type II irregular points can be significantly simplified by letting all the $u_{x}^{-}, u_{y}^{-}$equal zero. 
The level set function $\phi_{0}(x, y)$ of $\Gamma_{0}$ is given by

$$
\phi_{0}(x, y)= \begin{cases}-y-1 / 13 & x<-11 / 23 \\ -y-3(x+11 / 23) / 4-1 / 13 & x \geqslant-11 / 23 .\end{cases}
$$

The level set function $\phi_{i}(x, y)$ of $\Gamma_{i}$ is given by

$$
\phi_{i}(x, y)= \begin{cases}y+1 / 3 & x<-11 / 23, \\ y+3(x+11 / 23) / 4+1 / 3 & x \geqslant-11 / 23 .\end{cases}
$$

The exact solution is given by:

$$
\begin{aligned}
& u^{+}(x, y)=1+x y, \\
& u_{0}^{-}(x, y)=\cos (\pi x) \sin (\pi y), \\
& u_{i}^{-}(x, y)=0,
\end{aligned}
$$

with discontinuous coefficients

$$
\begin{aligned}
& \beta^{+}(x, y)=\frac{x^{2}-y^{2}+3}{7}, \\
& \beta_{0}^{-}(x, y)=\frac{x y+2}{5}, \\
& \beta_{i}^{-}(x, y)=0 .
\end{aligned}
$$

Numerical results in Table 8 verify the convergence of the proposed method.

\section{Case 7}

It is interesting to discuss a weak-solution problem whose solution has unbounded first order derivative on sharp edge [10]. Consider a domain $\Omega=\left[\begin{array}{ll}-1 & 1\end{array}\right] \times\left[\begin{array}{ll}-1 & 1\end{array}\right]$. Let us define

$$
\Omega^{-}=\left\{(x, y) \in \mathfrak{R}^{2} ; x^{2}+y^{2}<\frac{4}{9}, x<0 \text { or } y>0\right\},
$$

with reentrant corner (see Fig. 17). The solution on the domain $\Omega^{+}=\Omega \backslash \Omega^{-}$vanishes. The solution in $\Omega^{-}$is a harmonic function $u(x, y)=\operatorname{Im}\left(z^{2 / 3}\right)$, solving the boundary-value problem

$$
\begin{aligned}
& \Delta u=0 \quad \text { in } \Omega, \\
& u\left(e^{i \varphi}\right)=\frac{2}{3} \sin \left(\frac{2}{3} \varphi\right) \text { for } 0 \leqslant \varphi \leqslant \frac{3 \pi}{2}, \\
& u=0 \text { elsewhere on } \partial \Omega^{-} .
\end{aligned}
$$

The solution is $C^{2}(\Omega) \cap C^{0}\left(\overline{\Omega^{-}}\right)$. Finite element (Galerkin) formulations can handle this problem directly $[10,25]$. However, this problem cannot be directly solved by collocation formulations. A standard technique is to introduce an algebraic factor. Let us define a function

$$
w(z)=z^{\frac{8}{3}},
$$

where $w(z)$ satisfies $\Delta w=0$ with appropriate boundary condition. When $w(z)$ has been obtained from solving the harmonic equation, it is simply an algebraic operation to resolve the solution $u$

Table 8

Numerical accuracy tests of the thin layer case (Case 6)

\begin{tabular}{lll}
\hline$n_{x} \times n_{y}$ & $L_{\infty}$ & Order \\
\hline $20 \times 20$ & $1.54 \mathrm{e}-2$ & 1.71 \\
$40 \times 40$ & $4.70 \mathrm{e}-3$ & 2.88 \\
$80 \times 80$ & $6.36 \mathrm{e}-4$ & 2.01 \\
$160 \times 160$ & $1.58 \mathrm{e}-4$ & \\
\hline
\end{tabular}



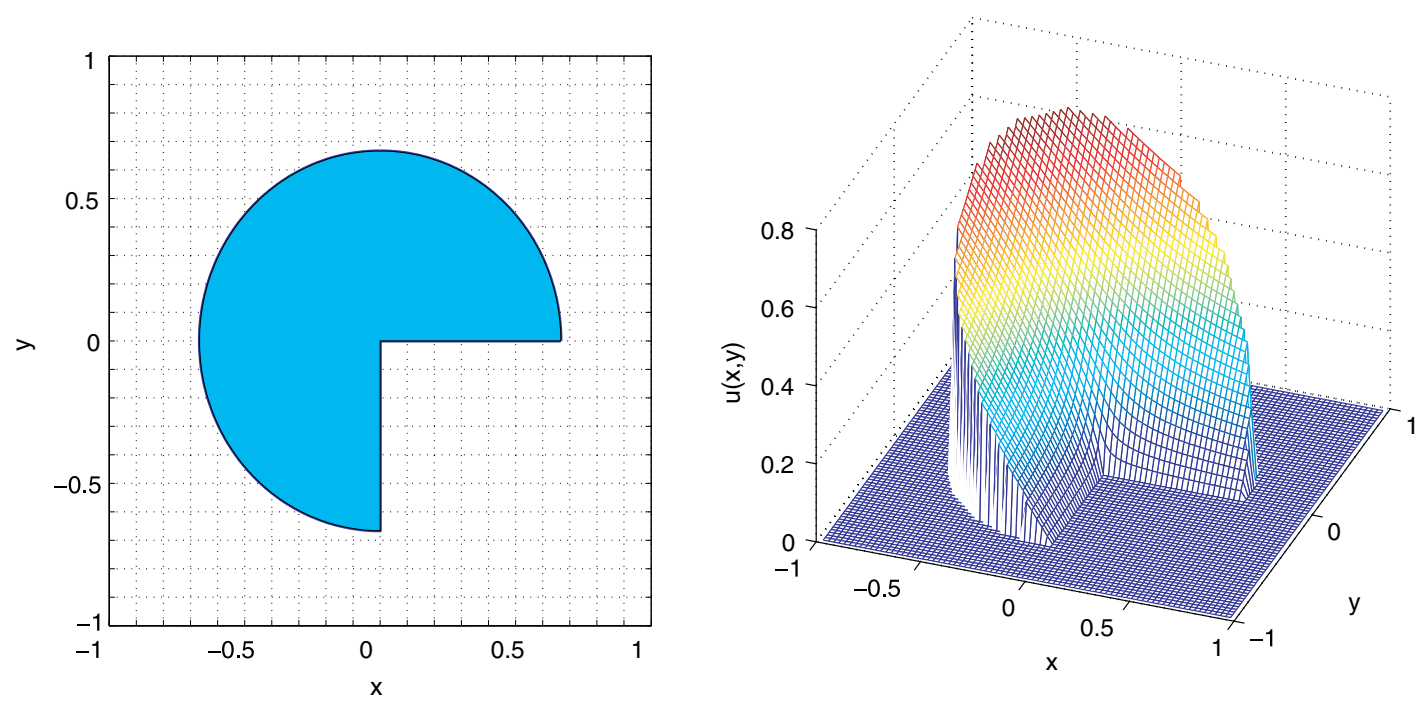

Fig. 17. Domain with reentrant corner on a $20 \times 20$ mesh (left) and computed solution (right) (Case 7).

$$
u(x, y)=\operatorname{Im}\left(z^{2 / 3}\right)= \begin{cases}\operatorname{Im}\left(w^{\frac{1}{4}}\right) & \text { for } 0 \leqslant \varphi<\frac{3 \pi}{4}, \\ \operatorname{Im}\left(\mathrm{i} w^{\frac{1}{4}}\right) & \text { for } \frac{3 \pi}{4} \leqslant \varphi<\frac{3 \pi}{2}, \\ \operatorname{Im}\left(-w^{\frac{1}{4}}\right) & \text { elsewhere. }\end{cases}
$$

Both the imaginary and real parts of function $w(z)$ are harmonic function, therefore they can be easily solved by the present MIB method. The errors of both the imaginary and real parts of function $w(z)$ are given in Table 9. Numerical results of $\operatorname{Im}(w)$ and $\operatorname{Re}(w)$ verify the convergence of the proposed method. The numerical solution of $u(x, y)$ obtained by Eq. (82) is shown in Fig. 17.

We next test accuracy and convergence on a sharp-edge case that has large contrast ratios of diffusion coefficients. The solution is $v=\operatorname{Re}(w(z))$ in $\Omega^{-}$and $v=0$ in $\Omega^{+}$, where $\Omega^{-}$and $\Omega^{+}$are defined above. Here, $v(x, y)$ satisfies $\nabla \cdot\left(\beta^{+} \nabla v\right)=0$ in $\Omega^{+}$and $\nabla \cdot\left(\beta^{-} \nabla v\right)=0$ in $\Omega^{-}$. We fixed the diffusion coefficient $\beta^{-}$to be 1 . The

Table 9

Numerical accuracy tests of the imaginary and real parts of $w(z)=z^{\frac{8}{3}}$ (Case 7)

\begin{tabular}{|c|c|c|c|c|}
\hline \multirow[t]{2}{*}{$n_{x} \times n_{y}$} & \multicolumn{2}{|l|}{$\operatorname{Im}(w(z))$} & \multicolumn{2}{|l|}{$\operatorname{Re}(w(z))$} \\
\hline & $L_{\infty}$ & Order & $L_{\infty}$ & Order \\
\hline $20 \times 20$ & $8.36 \mathrm{e}-4$ & & $1.06 \mathrm{e}-3$ & \\
\hline $40 \times 40$ & $2.36 \mathrm{e}-4$ & 1.81 & $2.02 \mathrm{e}-4$ & 2.39 \\
\hline $80 \times 80$ & $4.96 e-5$ & 2.26 & $3.54 \mathrm{e}-5$ & 2.51 \\
\hline $160 \times 160$ & $1.24 \mathrm{e}-5$ & 1.99 & $6.94 \mathrm{e}-6$ & 2.35 \\
\hline
\end{tabular}

Table 10

Numerical accuracy tests of large discontinuity ratio of $\beta^{-}$and $\beta^{+}$(Case 7)

\begin{tabular}{|c|c|c|c|c|}
\hline \multirow[t]{2}{*}{$\overline{n_{x} \times n_{y}}$} & \multicolumn{2}{|c|}{$\beta^{+}=10,000$} & \multicolumn{2}{|c|}{$\beta^{+}=0.001$} \\
\hline & $L_{\infty}$ & Order & $L_{\infty}$ & Order \\
\hline $20 \times 20$ & $8.00 \mathrm{e}-4$ & & $3.48 \mathrm{e}-3$ & \\
\hline $40 \times 40$ & $1.33 \mathrm{e}-4$ & 2.59 & $1.46 \mathrm{e}-3$ & 1.25 \\
\hline $80 \times 80$ & $2.19 \mathrm{e}-5$ & 2.60 & $2.67 \mathrm{e}-4$ & 2.45 \\
\hline $160 \times 160$ & $4.97 \mathrm{e}-6$ & 2.14 & $9.21 \mathrm{e}-5$ & 1.54 \\
\hline
\end{tabular}


numerical errors for $\beta^{+}=1$ have been given in columns 4 and 5 in Table 9. Table 10 shows the numerical errors for $\beta^{+}=10,000$ and $\beta^{+}=0.001$, respectively.

We found that the present method is not entirely robust with respect to large contrast ratios of diffusion coefficients. In the case $\beta^{+} \gg \beta^{-}$, the numerical solution converges faster than 2 nd order. While in the case $\beta^{+} \ll \beta^{-}$, the numerical solution converges slower than 2 nd order. This behavior will be explored further in our future work.

\section{Conclusion}

A wide variety of scientific and engineering problems involve sharp-edged material interfaces, and their governing equations are of elliptic type. These problems call for new efficient methods that do not depend on massive local mesh refinement, which does not work for highly oscillatory waves due to the pollution effect [3]. The present work provides a solution to this class of problems on the Cartesian grid by extending the marched interface and boundary (MIB) method [60,61] previously designed for straight or curved interfaces. The concept of secondary fictitious values is introduced to deal with difficult topology where primary fictitious values cannot be solved directly. The present MIB method is extensively validated by problems with sharpedged interfaces, as well as problems with a combination of sharp edges and oscillatory solutions, which pose a severe challenge to most existing numerical methods. The designed second order convergence has been confirmed for all the test problems, which has significantly improved the previous best result, 0.8 th order, reported by Hou and Liu [25] using a finite element formulation for similar problems.

The main ideas of the present MIB method are follows. First, simple Cartesian grids are used even if the problem is defined on an irregular domain and/or with sharp-edged interfaces. Second, the standard (higherorder) central finite difference (FD) schemes are utilized so that the condition number of the discretization matrix is relatively low, and efficient linear algebraic equation solvers can be used. Third, primary fictitious values are created on the irregular points near the interface to facilitate the use of the central FD schemes. Secondary fictitious values are introduced to resolved primary ones. Fourth, the fictitious values are determined by using physical jump conditions whose proper enforcement warrants the convergence of the FD discretization. Fifth, physical jump conditions are enforced "on the interface" to ensure an efficient restoration of accuracy. Sixth, the lowest order jump conditions are used to avoid the possible involvement of cross derivatives in the higher-order jump conditions and higher dimensional polynomials. Finally, to achieve higher-order convergence, the lowest order jump conditions are implemented repeatedly. The application of the present sharp-edged interface method is under our consideration to electromagnetic and acoustic wave scattering, fluid dynamics and aerodynamics.

\section{Acknowledgments}

This work was supported in part by NSF Grant IIS-0430987 and NSF Grant DMS-0616704.

\section{References}

[1] L. Adams, Z.L. Li, The immersed interface/multigrid methods for interface problems, SIAM J. Sci. Comput. 24 (2002) $463-479$.

[2] I. Babuška, The finite element method for elliptic equations with discontinuous coefficients, Computing 5 (1970) $207-213$.

[3] I. Babuška, S.A. Sauter, Is the pollution effect of the FEM avoidable for the Helmholtz equation considering high wave number? SIAM J. Numer. Anal. 34 (1997) 2392-2423.

[4] F. Baetke, H. Werner, H. Wengle, Numerical-simulation of turbulent-flow over surface-mounted obstacles with sharp edges and corners, J. Wind Engng. Indust. Aerodyn. 35 (1990) 129-147.

[5] G. Bao, G.W. Wei, S. Zhao, Numerical solution of the Helmholtz equation with high wave numbers, Int. J. Numer. Methods Engng. 59 (2004) 389-408.

[6] H. Ben Ameur, M. Burger, B. Hackl, Level set methods for geometric inverse problems in linear elasticity, Inverse Problems 20 (2004) 673-696.

[7] P.A. Berthelsen, A decomposed immersed interface method for variable coefficient elliptic equations with non-smooth and discontinuous solutions, J. Comput. Phys. 197 (2004) 364-386.

[8] G. Biros, L.X. Ying, D. Zorin, A fast solver for the Stokes equations with distributed forces in complex geometries, J. Comput. Phys. 193 (2004) 317-348. 
[9] J. Bramble, J. King, A finite element method for interface problems in domains with smooth boundaries and interfaces, Adv. Comput. Math. 6 (1996) 109-138.

[10] D. Braess, Finite Elements: Theory, Fast Solvers, and Applications in Solid Mechanics, Cambridge University Press, 1997.

[11] W. Cai, S.Z. Deng, An upwinding embedded boundary method for Maxwell's equations in media with material interfaces: 2D case, J. Comput. Phys. 190 (2003) 159-183.

[12] S. Caorsi, M. Pastorino, M. Raffetto, Electromagnetic scattering by a conducting strip with a multilayer elliptic dielectric coating, IEEE Trans. Electromag. Compat. 41 (1999) 335-343.

[13] Z.J. Cendes, D.N. Shenton, H. Shahnasser, Magnetic field computation using Delaney triangulation and complementary finite element methods, IEEE Trans. Magn. 19 (1983) 2251-2554.

[14] S.Z. Deng, K. Ito, Z.L. Li, Three-dimensional elliptic solvers for interface problems and applications, J. Comput. Phys. 184 (2003) 215-243.

[15] M.A. Dumett, J.P. Keener, An immersed interface method for solving anisotropic elliptic boundary value problems in three dimensions, SIAM J. Sci. Comput. 25 (2003) 348-367.

[16] E.A. Fadlun, R. Verzicco, P. Orlandi, J. Mohd-Yusof, Combined immersed-boundary finite-difference methods for three-dimensional complex flow simulations, J. Comput. Phys. 161 (2000) 30-60.

[17] R.P. Fedkiw, T. Aslam, B. Merriman, S. Osher, A non-oscillatory Eulerian approach to interfaces in multimaterial flows (the ghost fluid method), J. Comput. Phys. 152 (1999) 457-492.

[18] A.L. Fogelson, J.P. Keener, Immersed interface methods for Neumann and related problems in two and three dimensions, SIAM J. Sci. Comput. 22 (2000) 1630-1654.

[19] M. Francois, W. Shyy, Computations of drop dynamics with the immersed boundary method, Part 2: Drop impact and heat transfer, Numer. Heat Trans. Part B-Fund. 44 (2003) 119-143.

[20] F. Gibou, R.P. Fedkiw, A fourth order accurate discretization for the Laplace and heat equations on arbitrary domains, with applications to the Stefan problem, J. Comput. Phys. 202 (2005) 577-601.

[21] B.E. Griffith, C.S. Peskin, On the order of accuracy of the immersed boundary method: higher order convergence rates for sufficiently smooth problems, J. Comput. Phys. 208 (2005) 75-105.

[22] G. Guyomarch, C.-O. Lee, A discontinuous Galerkin method for elliptic interface problems with application to electroporation, AIST DAM Research Report, 04-14, 2004.

[23] G.R. Hadley, High-accuracy finite-difference equations for dielectric waveguide analysis I: uniform regions and dielectric interfaces, J. Lightw. Technol. 20 (2002) 1210-1218.

[24] J.S. Hesthaven, High-order accurate methods in time-domain computational electromagnetics. A review, Adv. Imaging Electron Phys. 127 (2003) 59-123.

[25] S. Hou, X.-D. Liu, A numerical method for solving variable coefficient elliptic equation with interfaces, J. Comput. Phys. 202 (2005) 411-445.

[26] T.Y. Hou, Z.L. Li, S. Osher, H. Zhao, A hybrid method for moving interface problems with application to the Hele-Shaw flow, J. Comput. Phys. 134 (1997) 236-252.

[27] H. Huang, Z.L. Li, Convergence analysis of the immersed interface method, IMA J. Numer. Anal. 19 (1999) $583-608$.

[28] J.K. Hunter, Z.L. Li, H. Zhao, Reactive autophobic spreading of drops, J. Comput. Phys. 183 (2002) 335-366.

[29] G. Iaccarino, R. Verzicco, Immersed boundary technique for turbulent flow simulations, Appl. Mech. Rev. 56 (2003) 331-347.

[30] S. Jin, X.L. Wang, Robust numerical simulation of porosity evolution in chemical vapor infiltration II. Two-dimensional anisotropic fronts, J. Comput. Phys. 179 (2002) 557-577.

[31] H. Johansen, P. Colella, A Cartesian grid embedding boundary method for Poisson's equation on irregular domains, J. Comput. Phys. 147 (1998) 60-85.

[32] J.D. Kandilarov, Immersed interface method for a reaction-diffusion equation with a moving own concentrated source, Lecture Notes Comput. Sci. 2542 (2003) 506-513.

[33] M.C. Lai, C.S. Peskin, An immersed boundary method with formal second-order accuracy and reduced numerical viscosity, J. Comput. Phys. 160 (2000) 705-719.

[34] L. Lee, R.J. LeVeque, An immersed interface method for incompressible Navier-Stokes equations, SIAM J. Sci. Comput. 25 (2003) $832-856$.

[35] R.J. LeVeque, Z.L. Li, The immersed interface method for elliptic equations with discontinuous coefficients and singular sources, SIAM J. Numer. Anal. 31 (1994) 1019-1044.

[36] Z.L. Li, A fast iterative algorithm for elliptic interface problems, SIAM J. Numer. Anal. 35 (1998) $230-254$.

[37] Z.L. Li, K. Ito, Maximum principle preserving schemes for interface problems with discontinuous coefficients, SIAM J. Sci. Comput. 23 (2001) 339-361.

[38] Z.L. Li, T. Lin, X.H. Wu, New Cartesian grid methods for interface problems using the finite element formulation, Numer. Math. 96 (2003) 61-98.

[39] Z.L. Li, S.R. Lubkin, Numerical analysis of interfacial two-dimensional Stokes flow with discontinuous viscosity and variable surface tension, Int. J. Numer. Methods Fluid 37 (2001) 525-540.

[40] Z.L. Li, W.-C. Wang, I.-L. Chern, M.-C. Lai, New formulations for interface problems in polar coordinates, SIAM J. Sci. Comput. 25 (2003) 224-245.

[41] M.N. Linnick, H.F. Fasel, A high-order immersed interface method for simulating unsteady incompressible flows on irregular domains, J. Comput. Phys. 204 (2005) 157-192. 
[42] B. Lombard, J. Piraux, How to incorporate the spring-mass conditions in finite-difference schemes, SIAM J. Sci. Comput. 24 (2003) 1379-1407.

[43] E.B. Macak, W.D. Munz, J.M. Rodenburg, Plasma-surface interaction at sharp edges and corners during ion-assisted physical vapor deposition. Part I: Edge-related effects and their influence on coating morphology and composition, J. Appl. Phys. 94 (2003) 28292836.

[44] A. Mayo, The fast solution of Poisson's and the biharmonic equations on irregular regions, SIAM J. Numer. Anal. 21 (1984) $285-299$.

[45] A. Mckenney, L. Greengard, A. Mayo, A fast Poisson solver for complex geometries, J. Comput. Phys. 118 (1995) $348-355$.

[46] R. Miniowitz, J.P. Webb, Covariant-projection quadrilateral elements for the analysis of wave-guides with sharp edges, IEEE Trans. Microwave Theory Techn. 39 (1991) 501-505.

[47] R. Mittal, G. Iaccarino, Immersed boundary methods, Annu. Rev. Fluid Mech. 37 (2005) 236-261.

[48] G. Pan, M. Tong, B. Gilbert, Multiwavelet based moment method under discrete Sobolev-type norm, Microwave Opt. Techn. Lett. 40 (2004) 47-50.

[49] Z. Pantic-Tanner, J.Z. Savage, D.R. Tanner, A.F. Peterson, Two-dimensional singular vector elements for finite-element analysis, IEEE Trans. Microwave Theory Techn. 46 (1998) 178-184.

[50] C.S. Peskin, Numerical analysis of blood flow in heart, J. Comput. Phys. 25 (1977) 220-252.

[51] C.S. Peskin, Lectures on mathematical aspects of physiology, Lect. Appl. Math. 19 (1981) 69-107.

[52] C.S. Peskin, D.M. Mcqueen, A 3-dimensional computational method for blood-flow in the heart. 1. Immersed elastic fibers in a viscous incompressible fluid, J. Comput. Phys. 81 (1989) 372-405.

[53] M. Schulz, G. Steinebach, Two-dimensional modelling of the river Rhine, J. Comput. Appl. Math. 145 (2002) 11-20.

[54] J.A. Sethian, A. Wiegmann, Structural boundary design via level set and immersed interface methods, J. Comput. Phys. 163 (2000) 489-528.

[55] A.K. Tornberg, B. Engquist, Numerical approximations of singular source terms in differential equations, J. Comput. Phys. 200 (2004) 462-488.

[56] B.J.E. van Rens, W.A.M. Brekelmans, F.P.T. Baaijens, Modelling friction near sharp edges using a Eulerian reference frame: application to aluminum extrusion, Int. J. Numer. Methods Engng. 54 (2002) 453-471.

[57] J.V. Voorde, J. Vierendeels, E. Dick, Flow simulations in rotary volumetric pumps and compressors with the fictitious domain method, J. Comput. Appl. Math. 168 (2004) 491-499.

[58] J.H. Walther, G. Morgenthal, An immersed interface method for the vortex-in-cell algorithm, J. Turbulence 3 (2002). Art. No. 039.

[59] A. Wiegmann, K.P. Bube, The explicit-jump immersed interface method: finite difference methods for PDEs with piecewise smooth solutions, SIAM J. Numer. Anal. 37 (2000) 827-862.

[60] S. Zhao, G.W. Wei, High order FDTD methods via derivative matching for Maxwell's equations with material interfaces, J. Comput. Phys. 200 (2004) 60-103.

[61] Y.C. Zhou, S. Zhao, M. Feig, G.W. Wei, High order matched interface and boundary (MIB) schemes for elliptic equations with discontinuous coefficients and singular sources, J. Comput. Phys. 231 (2006) 1-30.

[62] Y.C. Zhou, G.W. Wei, On the fictitious-domain and interpolation formulations of the matched interface and boundary (MIB) method, J. Comput. Phys. 219 (2006) 228-246. 